\title{
Approaches for Sample Characterization and Lithography with Nanoparticles using Modes of Scanning Probe Microscopy
}

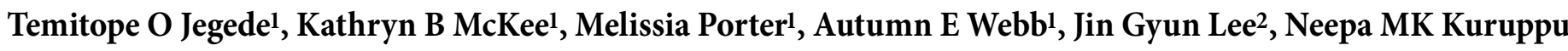 \\ Arachchigel, Justin R Ragains ${ }^{1}$, Noémie Elgrishi ${ }^{1}$ and Jayne C Garno ${ }^{\star}$ \\ ${ }^{1}$ Department of Chemistry, Louisiana State University, Baton Rouge, LA 70803, USA \\ ${ }^{2}$ Cain Department of Chemical Engineering, Louisiana State University, Baton Rouge, LA 70803, USA
}

\section{Article Info}

\author{
*Corresponding author: \\ Jayne C Garno \\ Department of Chemistry \\ Louisiana State University \\ 232 Choppin Hall \\ Baton Rouge, LA 70803 \\ Tel: 225-578-8942 \\ Fax: 225-5783458 \\ E-mail: jgarno@lsu.edu
}

Received: May 17, 2019

Accepted: June 3, 2019

Published: June 10, 2019

Citation: Jegede TO, McKee KB, Porter M, et al. Approaches for Sample Characterization and Lithography with Nanoparticles using Modes of Scanning Probe Microscopy. Madridge J Anal Sci Instrum. 2019; 4(1): 96-108.

doi: $10.18689 /$ mjai-1000119

Copyright: @ 2019 The Author(s). This work is licensed under a Creative Commons Attribution 4.0 International License, which permits unrestricted use, distribution, and reproduction in any medium, provided the original work is properly cited.

Published by Madridge Publishers

\begin{abstract}
Measurement and imaging modes of scanning probe microscopy (SPM) have been routinely applied for characterizing systems of nanoparticles; however the evolution of fabrication methods to prepare arrangements of nanoparticles remains a challenge. Reproducible fabrication of surface structures which integrate nanoparticles within ultra-small patterns will require innovative approaches to achieve high throughput and precision. Strategies for nanoscale lithography have been introduced for preparing defined arrangements of nanoparticles on surfaces based on physical or chemical interactions. For example, physisorption was employed for attaching nanoparticles based on colloidal lithography and site-directed assembly. Microfabricated atomic force microscope (AFM) tips with capillary channels have been used to pattern nanoparticles through electrostatic interactions. Specific chemical interactions can be designed for patterning nanoparticles with dip-pen nanolithography and SPM-based fabrication. Studies with nanoparticles are reviewed, which have applied either in situ and ex situ approaches for imaging and measurements using modes of SPM. The imaging principle for contact and tapping modes are described with example studies of nanoparticle patterns. The SPM modes for measuring physical properties (e.g. magnetism, softness, conductance) using force modulation microscopy (FMM), magnetic force microscopy (MFM), magnetic sample modulation (MSM), and conductive probe AFM are described for selected studies of lithography with nanoparticles. Strategies for patterning nanoparticles using lithography modes of nanoshaving, dip-pen nanolithography, and tip-induced oxidation have been reported for a range of nanoparticle systems. Applications for nanotechnology will require the integration of nanoparticles within engineered surface architectures. Stable, organized arrangements of nanoparticles with robust chemical/physical attachment to surfaces will be needed for applications, to fully gain advantages of the characteristic quantum properties of nanoparticles.
\end{abstract}

Keywords: Nanoparticles; Nanopatterns; SPM modes; Colloidal lithography; Scanning probe lithography; Nanofabrication; Nanodispensing.

\section{Introduction}

Measurement and imaging modes of scanning probe microscopy (SPM) provide capabilities for ultrasensitive characterizations of samples with exceptional resolution at the level of individual molecules or atoms. For studies with nanoparticles, several measurement and fabrication modes of SPM have been applied to acquire fundamental chemical and physical information, beyond basic characterizations of the size and shape 
of the samples. To incorporate nanoparticles within devices, directed assembly and patterning strategies have been developed to prepare defined arrangements on surfaces. To precisely assemble and position nanoparticles with control at the nanometer level remains a challenge and will require innovation for nanomaterials-based technology.

The optical, electrical, magnetic and catalytic properties of diverse systems of nanoparticles are different from that of bulk materials, which is attributable to quantum-scale dimensions [1]. The composition and chemistry of nanoparticles have been proposed for emerging technologies for sensors and photoelectronic devices [2]. Patterned arrangements of metal and semiconductor nanoparticles have been integrated in electronic circuits [3,4], single-electron tunneling devices [5], electronic connections and 3D plasmonic structures [6]. Surface patterns of nanoparticles have been tested as substrates for surface enhanced Raman spectroscopy (SERS) substrates $[7,8]$.

Strategies have been developed to chemically attach nanoparticles in designed arrangements, which can help to address problems with SPM characterizations as well as to enable production of complex structures for technical applications. To define the surface placement of nanoparticles, combining nanoscale lithography with SPM measurements is a practical strategy to enable well-resolved surface characterizations with systems of nanoparticles.

To accomplish lithography, parameters which influence the localization of nanoparticles to form well-defined patterns include the chemical nature of the nanoparticle as well as the reactivity of the interface. The interface can be designed to integrate chemical specificity for binding nanoparticles using approaches with chemistry-based lithography. The hydrophobic or hydrophilic nature of the substrate is a key parameter for accomplishing nanoscale lithography. In this report, we review lithographic approaches with nanoparticles and discuss strategies for surface characterization using modes of SPM. The strategies for nanopatterning are determined by the complex interplay of the chemical/physical nature of the substrate, the surface composition of the nanoparticle as well as the selection of organic molecules used as linkers.

\section{Characterization of Nanoparticle Patterns with Modes of SPM}

For SPM imaging, the shapes of surface features are outlined by a sharp probe, with an apex which is often greater in size than the dimensions of samples. The resolution that is typically achieved for SPM is influenced by the nature of the sample as well as instrument parameters. Studies using modes of SPM with nanoparticles can be challenging because the adhesive interactions between the tip and sample can displace and push around materials that are weakly adsorbed at the solid interface. Further, when suspensions of nanoparticles are dried on flat surfaces the materials tend to deposit to form dense aggregates and multilayers. The individual shapes of features cannot be fully outlined for aggregates and clusters, because the scanning probe cannot penetrate between nanoparticles to trace the contour of nanoparticles that are closely packed together. Beyond imaging, information of physical properties such as friction, stiffness, adhesion, conductance, and magnetism can be obtained for nanoparticles using modes of SPM. Commercial SPM systems can be configured for physical measurements by changing certain instrument factors such as the nature of the probe (magnetic, conductive) or the operational parameters for feedback. For measurement modes, the probe is used as a force sensor to measure interactions between the tip and sample at scales from nanometers to microns. The modes listed in table 1 have been applied for characterizing nanoparticles and will be successively described, followed by examples from previous reports.

Table 1. Measurement and Imaging modes of SPM Appliedfor Studies with Nanoparticles [9-13].

\begin{tabular}{|c|c|c|c|c|}
\hline Mode & \begin{tabular}{|l|} 
Resolution \\
Achieved*
\end{tabular} & Type of probe & \begin{tabular}{|l|} 
Imaging \\
Environment
\end{tabular} & Measurements \\
\hline Contact mode [9] & $<1 \mathrm{~nm}$ & $\begin{array}{l}\text { soft probes } \\
\mathrm{Si}_{1} \mathrm{Si}_{3} \mathrm{~N}_{4}\end{array}$ & liquid, air & $\begin{array}{l}\text { topography } \\
\text { friction }\end{array}$ \\
\hline Tapping mode [9] & $\sim 1 \mathrm{~nm}$ & $\begin{array}{l}\text { hard probes } \\
\mathrm{Si}, \mathrm{Si}_{3} \mathrm{~N}_{4}\end{array}$ & air & $\begin{array}{l}\text { topography } \\
\text { elastic response }\end{array}$ \\
\hline $\begin{array}{l}\text { Force Modulation } \\
\text { Microscopy (FMM) } \\
{[10]}\end{array}$ & $<2 \mathrm{~nm}$ & $\begin{array}{l}\text { hard or soft } \\
\text { probes, } \mathrm{Si}_{1} \mathrm{Si}_{3} \mathrm{~N}_{4}\end{array}$ & air, liquid & $\begin{array}{l}\text { topography } \\
\text { amplitude phase }\end{array}$ \\
\hline $\begin{array}{l}\text { Magnetic Sample } \\
\text { Modulation (MSM) } \\
{[11]}\end{array}$ & $<1 \mathrm{~nm}$ & $\begin{array}{l}\text { soft, } \\
\text { nonmagnetic } \\
\text { probes, } \mathrm{Si}_{1}, \mathrm{Si}_{3} \mathrm{~N}_{4}\end{array}$ & air & $\begin{array}{l}\text { topography } \\
\text { amplitude phase }\end{array}$ \\
\hline $\begin{array}{l}\text { Conductive Probe } \\
\text { AFM [12] }\end{array}$ & $<1 \mathrm{~nm}$ & $\begin{array}{l}\text { metal coated } \\
\text { probe } \\
\text { (underside) }\end{array}$ & air & $\begin{array}{l}\text { topography } \\
\text { current images } \\
\text { I-V spectra }\end{array}$ \\
\hline $\begin{array}{l}\text { Magnetic Force } \\
\text { Microscopy (MFM) } \\
{[13]}\end{array}$ & $200 \mathrm{~nm}^{* *}$ & $\begin{array}{l}\text { magnetic coating } \\
\text { on probe } \\
\text { (underside) }\end{array}$ & air & $\begin{array}{l}\text { surface maps of } \\
\text { magnetic forces }\end{array}$ \\
\hline
\end{tabular}

*The resolution achieved is affected by the nature of the sample and the aspect ratio of the probe.

**Typical resolution is around $200 \mathrm{~nm}$, unless a specialized sharp probe is used for MFM.

\section{Imaging Nanoparticles with Contact and Tapping Modes of SPM}

For operation in contact mode, the tip is placed in continuous contact with the surface under a certain force, as the probe is scanned across the sample in a raster pattern. As the tip moves up or down across the sample during scans, changes in the deflection of the cantilever are detected with a photodetector as outlined in figure 1a. Images of topography are generated point-by-point to map changes in tip deflection compared to an initial force set point. The electronic controllers for SPM instruments enable precise adjustments for the $z$ direction of the scanner using a feedback loop to control the amount of force applied to the tip. Contact mode atomic force microscopy (AFM) enables ultrasensitive 3D measurements, to achieve lateral resolution of $0.1 \mathrm{~nm}$ and vertical resolution of $0.01 \mathrm{~nm}$ [14]. Using contact mode AFM, atomic resolution has been achieved with a sharp tip for ultraflat surfaces such as graphite [15], molybdenum sulfide [16], inorganic crystals [17,18] and metal oxides [19].

The shear forces and stick-slip adhesion of a probe operated in contact mode can cause perturbation of the surface or may damage soft or fragile samples. Achieving well-resolved images can be problematic if the tip sticks to 
the surface, producing line spikes in images. Tapping mode was developed to overcome stick-slip adhesion, in which the tip is operated in intermittent contact to improve resolution for imaging delicate or soft and sticky samples. Tapping mode can also be helpful for preventing the probe from dislodging or pushing nanoparticles from the substrate.

For tapping mode operation (Figure 1b), the AFM cantilever is driven by a piezo actuator to rapidly "tap" the surface by oscillation near the resonance frequency. The tip is placed sufficiently close to the surface such that it is intermittently driven to make contact and then disengage. A small piezo-actuator element is used to drive the tapping motion of the cantilever. The tip is driven to quickly touch the surface to prevent transient interfacial binding of the probe to the surface to mitigate problems caused by stick-slip adhesion. The feedback loop is configured to maintain a constant amplitude of the cantilever motion compared to the initial driving signal. As the tapping tip is scanned across a sample, the oscillation of the cantilever is affected by tipsurface interactions (damping effects) which can be sensitively detected using a lock-in amplifier. With tapping mode, adjustments of the voltages are converted to digital images to generate topography and phase frames which are acquired concurrently. Shifts in the amplitude and phase of the cantilever are plotted to obtain information of the elastic response of nanomaterials.

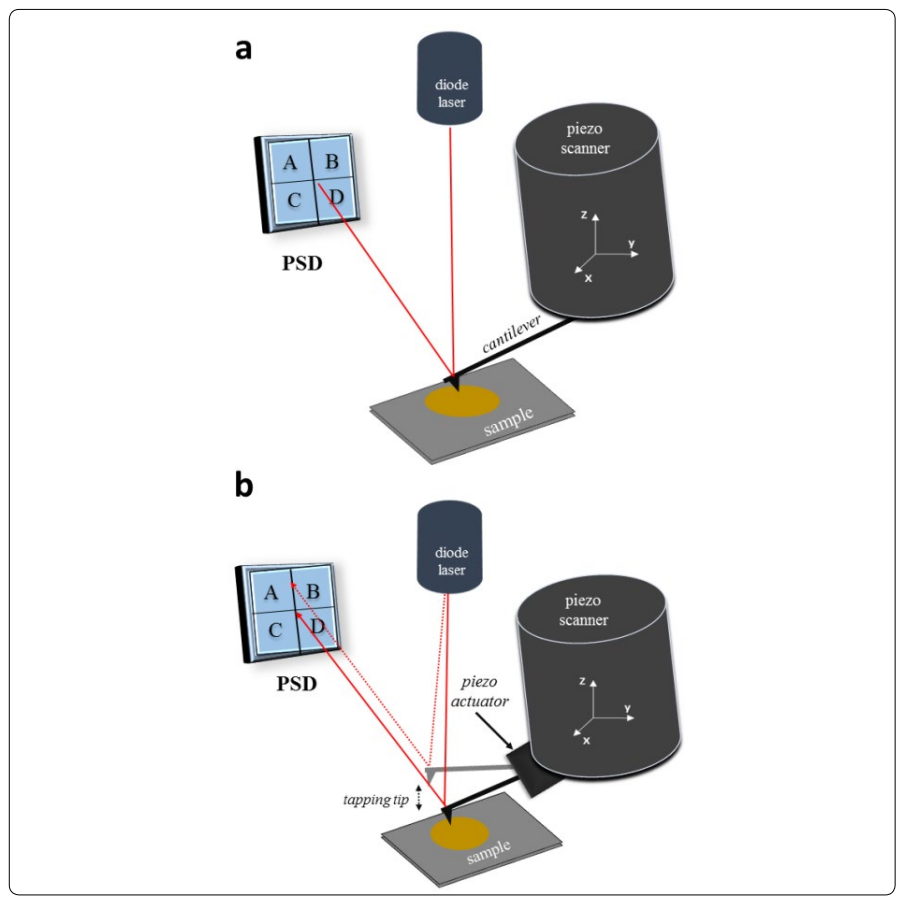

Figure 1. Instrument diagram for (a) contact mode and (b) tapping-mode AFM.

\section{Characterization of Nanoparticles with Force Modulation Microscopy (FMM)}

Force modulation microscopy (FMM) is used for studying the mechanical characteristics of samples, as introduced by Maivald et al. in 1991 [20]. Information of elastic and viscoelastic $[21,22]$ properties as well as quantitative measurements of Young's modulus can be measured at the nanoscale [13,23]. For
FMM operation, a feedback loop is used to maintain a constant cantilever deflection during imaging to generate topography frames, in the same configuration as contact-mode AFM. During scans, the sample is driven to vibrate in the $z$ direction by a piezo actuator placed under the sample (Figure 2a). Unlike tapping mode, the tip remains in continuous contact with the vibrating sample for FMM imaging. With FMM, the force between the tip and sample is modulated which causes shifts in the amplitude and phase vibration of the probe/sample compared to the driving AC signal. As the tip is traced over a hard surface, the amplitude response increases in magnitude compared to the driving signal (Figure $2 b$ ). Whereas, when the tip is scanned across a soft region, the amplitude is reduced because the oscillation is absorbed or dampened. The amplitude and phase shifts of the signal from a position sensitive detector (PSD) are mapped concurrently with topography frames to generate digital images from the amplitude and phase response. Local differences in the elastic response of the sample can be sensitively mapped to reveal differences in sample composition correspondingly with topography frames.

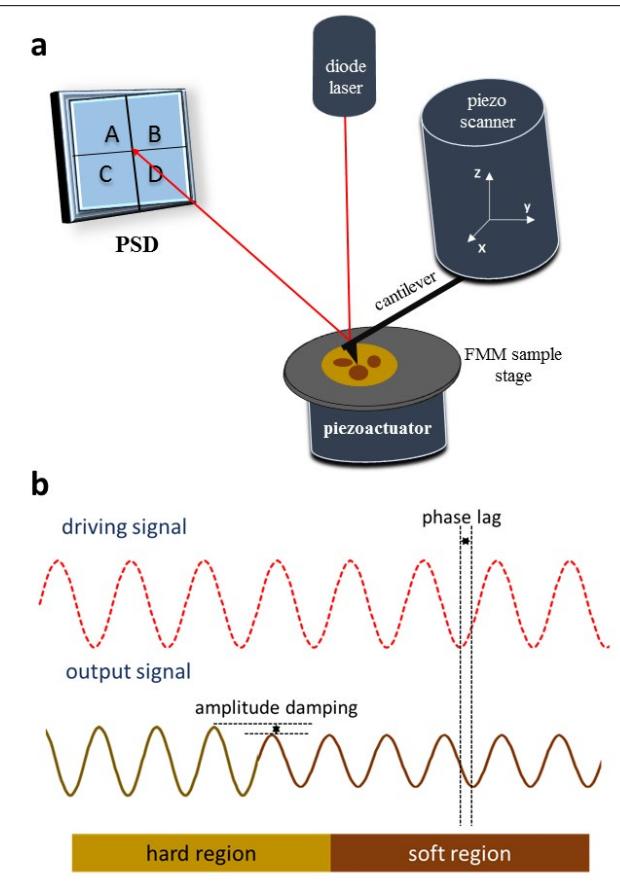

Figure 2. Operating principle for force modulation microscopy (FMM). (a) Instrument diagram; (b) Illustration of the changes in the phase and amplitude of tip motion for hard and soft samples.

\section{Measurements with Magnetic Force Microscopy (MFM) for Nanoparticle Samples}

The mode of magnetic force microscopy (MFM) uses a probe that is coated with a thin magnetic film of $\mathrm{Co}$, Fe or an alloy for operation in noncontact mode $[24,25]$. For the MFM configuration, a magnetic tip is lifted above the surface at discrete distances to sense the relatively weak, long-range forces of magnetic areas of samples. For MFM, the magnetic strength of the sample must be sufficiently strong to deflect or attract a micrometer-sized cantilever. For MFM operation in lift mode, the tip is scanned in contact mode to obtain a 
profile of the topography line-by-line, then lifted a certain distance to retrace the memorized surface corrugation during noncontact operation. During lift mode operation, the MFM tip will deflect according to the magnetic polarity of the sample to furnish surface maps of the strength and polarity of magnetic domains. The magnetic probe of MFM is used as a sensor to detect the relatively weak long-range forces of magnetic areas of surfaces operating at selected distances from 50-200 nm. The underside of the tip is coated with a magnetic metal which results in a fairly blunt probe, which limits the resolution of MFM to around $200 \mathrm{~nm}$. When an ultra-sharp specialized probe is used for MFM, resolution on the order of $5 \mathrm{~nm}$ has been reported [26]. The MFM mode has been used for measurements with surface arrangements of ferromagnetic nanoparticles composed of $\mathrm{Fe} / \mathrm{Co}$ [27], Fe/Pt [28], and $\mathrm{Fe} / \mathrm{Co} / \mathrm{P}$ [29], as well as for superparamagnetic magnetite nanoparticles [30].

\section{Investigations with Magnetic Sample Modulation AFM for Patterns of Nanoparticles}

Magnetic sample modulation (MSM) AFM is a hybrid of contact mode AFM combined with actuation of magnetic samples. The MSM mode has been applied for studies of magnetic materials such as ferritin [31], iron nanoclusters [32,33] and composite $\mathrm{FeNi}_{3}$ nanoparticles [11]. A nonmagnetic probe is required for MSM-AFM for operation using a force set point for feed back with contact mode configuration. When an AC electromagnetic field is applied to samples, the magnetic areas of the sample are driven to vibrate in response to the magnetic flux. A key variable for the imaging strategy for MSM is that a magnetic tip is not required. Instead of measuring magnetism directly, the sample vibration in response to the magnetic field is detected by changes in the motion of a non-magnetic probe placed in contact with the sample (Figure 3).

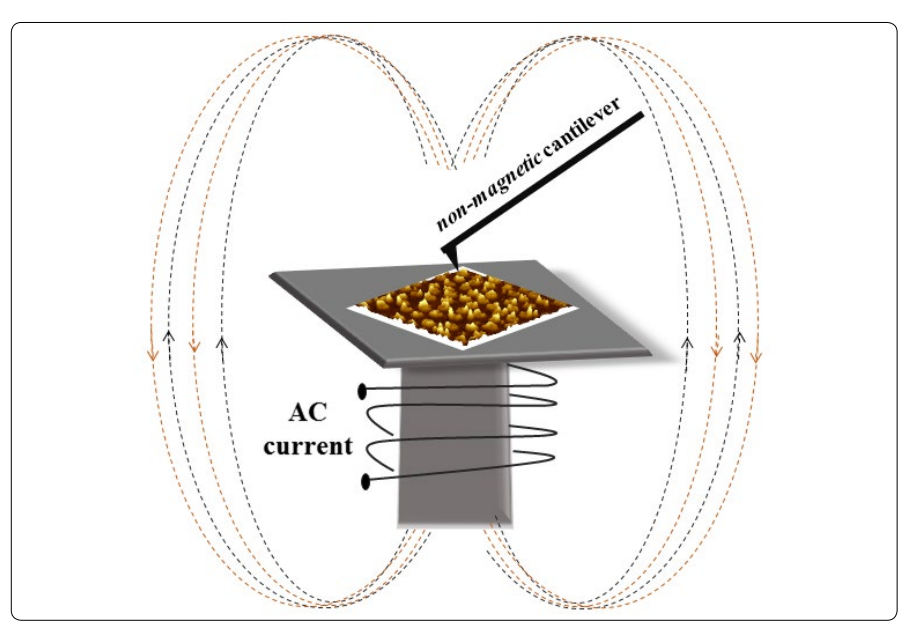

Figure 3. Instrument set-up for magnetic sample modulation, MSM-AFM, in which the sample is driven to vibrate by an AC electromagnetic field.

A solenoid placed under the sample is used to generate a magnetic field which alternates in polarity and strength according to the frequency and amplitude parameters of the
AC driving current. Samples are placed close to one end of the solenoid to experience the strongest flux of the field. Magnetic nanostructures are induced to vibrate in the electromagnetic field providing selective contrast for the magnetic areas that are in motion. The changes in MSM phase and amplitude relative to the driving $A C$ signal are detected and mapped with the MSM to identify the areas of magnetic domains.

With MSM-AFM, extreme sensitivity is achieved for slight changes in tip movement because a lock-in amplifier is used to acquire the amplitude and phase components of the deflection signals. The field strength applied for MSM is on the order of 0.05 to 0.6 Tesla, and high-field permanent magnets are not required for measurements. For the instrument set-up of MSM, positional feedback employs the force-deflection settings typical for contact mode to acquire topographic data. The MSM-AFM mode furnishes sensitive maps of the magnetic areas of samples. To obtain amplitude and phase components of the tip motion, an auxiliary output channel from the quadrant photodiode of the AFM scanner is directed to input channels of a lock-in amplifier, using the driving $\mathrm{AC}$ waveform as a reference. Thus, the periodic motion of the sample vibration can be tracked by changes in the deflection of the tip. The changes in phase angle and amplitude are plotted as a function of tip position to generate MSM phase and amplitude images.

\section{Characterization of Nanoparticle Samples with Conductive Probe AFM}

Conductive-probe AFM (CP-AFM) has been used for studies of the conductive properties of nanoparticles composed of gold [4,5,34], Pt [3], silica [35], and Ag [35]. Nanoparticles of noble metals ( $\mathrm{Pt}, \mathrm{Au}, \mathrm{Ag}$ ) were used as contacts for CP-AFM measurements between conducting polymers and metal nanoparticles reported by Cho and Park [3]. With the CP-AFM mode, topographic maps are acquired simultaneously with current images by applying a bias between a metal-coated tip and the sample. By parking the tip on individual nanoparticles or nanostructures, current-voltage (I-V) spectra can be acquired at local points by ramping the voltage. Standard AFM tips made of silicon or silicon nitride are coated with a thin layer (15-100 nm) of metal (Au, Pt, Co/Cr). To acquire I-V spectra, a bias voltage is applied to the sample, and the current through the tip-nanoparticle-sample junction is measured with a sensitive preamp placed near the tip. Conductive (gold and graphite) or semi-conductive (silicon) substrates are used for measurements with CP-AFM. Current maps can be acquired by scanning the tip in contact mode by applying a fixed bias to the sample.

\section{Approaches for Nanoscale Lithography with Nanoparticles}

A number of approaches have been developed for directing the assembly of nanoparticles on flat substrates to facilitate SPM measurements or to integrate nanoparticles in device designs. Chemistry-based patterning strategies for nanoparticles 
have been developed by functionalizing either the substrate or nanoparticles with specific groups to direct surface binding in defined arrangements. Physical adsorption has also been applied for patterning nanoparticles by dispensing solutions of particles which are attached to surfaces by physisorption when dried. Examples of techniques used for patterning nanoparticles at nanometer scale are summarized in table 2, with information of the SPM modes used for characterizing samples. In several examples, the SPM instrument was configured for both nanoscale lithography and imaging of nanoparticle patterns. Electronic and magnetic fields have been used for directing the assembly of magnetic nanoparticles in defined arrangements [36,37]. Techniques that have been used for high-throughput patterning with nanoparticles include dip-pen nanolithography [38,39], microcontact printing [40,41], and colloidal lithography $[1,42,43]$.

Table 2. Overview of the Protocols Applied for Patterning and Characterizing Nanoparticles [44-52].

\begin{tabular}{|c|c|c|c|c|}
\hline Nanolithography Approach & $\begin{array}{l}\text { Type \& Size of } \\
\text { Nanoparticles }\end{array}$ & \begin{tabular}{|l|} 
Feature Size \\
and Substrate
\end{tabular} & $\begin{array}{l}\text { Immobilization } \\
\text { Chemistry }\end{array}$ & SPM Modes \\
\hline Nanodispensing [44] & polystyrene, $25 \mathrm{~nm}$ & $\begin{array}{l}\text { spots, } 50 \mathrm{~nm} \\
\text { on APTES coated } \mathrm{SiO}_{2}\end{array}$ & electrostatic interactions & $\begin{array}{l}\text { contact mode for dispensing, tapping } \\
\text { mode AFM for imaging }\end{array}$ \\
\hline Nanodispensing [45] & gold, $15 \mathrm{~nm}$ & $\begin{array}{l}\text { arrays of spots, } 50 \mathrm{~nm} \text { to } \\
\text { microns on amino- } \mathrm{SiO}_{2}\end{array}$ & $\begin{array}{l}\text { diffusion, electrostatic } \\
\text { interactions }\end{array}$ & $\begin{array}{l}\text { contact mode for dispensing, tapping } \\
\text { mode AFM for imaging }\end{array}$ \\
\hline Nanodispensing [46] & $\begin{array}{l}\text { nanodiamonds } \\
4-10 \mathrm{~nm}\end{array}$ & $\begin{array}{l}\text { dot patterns, } 50-350 \mathrm{~nm} \\
\text { on glass }\end{array}$ & electrostatic interactions & $\begin{array}{l}\text { contact mode for dispensing, tapping } \\
\text { mode AFM for imaging }\end{array}$ \\
\hline SPM-based lithography [52] & $\begin{array}{l}\text { gold nanoparticles, } \\
13,30 \mathrm{~nm} \\
\text { gold nanorods, } 80 \times 20 \mathrm{~nm}\end{array}$ & $\begin{array}{l}100 \mathrm{~nm} \text { grooves on APTES } \\
\text { coated } \mathrm{SiO}_{2} / \mathrm{Si} \text { substrate }\end{array}$ & $\begin{array}{l}\text { electrostatic interactions } \\
\text { heated annealing }\end{array}$ & $\begin{array}{l}\text { contact mode for patterning, tapping mode } \\
\text { for imaging }\end{array}$ \\
\hline $\begin{array}{l}\text { Dip-Pen Nanolithography } \\
\text { (DPN) [39] }\end{array}$ & $\begin{array}{l}\text { gold nanoparticles } \\
1.4 \text { and } 5 \mathrm{~nm}\end{array}$ & $\begin{array}{l}\text { single dots and lines on } \\
\text { UVO-treatedSiO }\end{array}$ & electr & $\begin{array}{l}\text { contact mode for patterning, tapping mode } \\
\text { for imaging }\end{array}$ \\
\hline Nanoshaving [51] & $\begin{array}{l}\text { gold nanoparticles } \\
4-8 \mathrm{~nm}\end{array}$ & $\begin{array}{l}\text { rectangle, } 150 \times 450 \mathrm{~nm}^{2} \\
\text { within an alkanethiol SAM }\end{array}$ & chemisorption & $\begin{array}{l}\text { Contact mode for patterning and FMM } \\
\text { mode for imaging }\end{array}$ \\
\hline Site-directed synthesis [47] & gold nanoparticles, 30 nm & $\begin{array}{l}\text { dot patterns written with DPN } \\
\text { on silicon substrates }\end{array}$ & $\begin{array}{l}\text { diffusion from a coated } \\
\text { probe, then heated } \\
\text { annealing }\end{array}$ & contact mode AFM \\
\hline Site-directed synthesis [9] & $\begin{array}{l}\text { rare earth oxide } \\
\text { nanoparticles, } 90 \mathrm{~nm}\end{array}$ & $\begin{array}{l}\text { hexagonal arrays of individual } \\
\text { nanoparticles on } \mathrm{Si}(111)\end{array}$ & physisorption & tapping-mode AFM \\
\hline $\begin{array}{l}\text { Chemically driven assembly } \\
{[12]}\end{array}$ & gold, $18 \mathrm{~nm}$ & rings, arrays on silicon wafers & $\begin{array}{l}\text { electrostatic interactions } \\
\text { on oxidized OTS }\end{array}$ & $\begin{array}{l}\text { local probe oxidation for patterning, } \\
\text { tapping modeAFM for imaging }\end{array}$ \\
\hline $\begin{array}{l}\text { Chemically driven assembly } \\
\text { [48] }\end{array}$ & $\begin{array}{l}\text { gold nanoparticles } \\
2-6 \mathrm{~nm}\end{array}$ & $\begin{array}{l}\text { circular arrangements of } \\
\text { tightly-packed nanoparticles on } \\
\text { silicon wafers }\end{array}$ & electrostatic interactions & $\begin{array}{l}\text { local probe oxidation for patterning, } \\
\text { tapping modeAFM for imaging }\end{array}$ \\
\hline Nanosphere lithography[49] & $\begin{array}{l}\mathrm{TiO}_{2} \text { nanoparticles } \\
130-180 \mathrm{~nm}\end{array}$ & $\begin{array}{l}\text { hexagonal arrays } \\
\text { with } 420 \mathrm{~nm} \text { periodicity on } \\
\text { silicon substrates }\end{array}$ & $\begin{array}{l}\text { heated evaporation } \\
\text { of Ti ribbon }\end{array}$ & contact mode AFM for imaging \\
\hline Particle lithography[50] & $\begin{array}{l}\text { cysteine-coated CdS } \\
\text { nanoparticles, } 2 \mathrm{~nm}\end{array}$ & $\begin{array}{l}\text { rings of nanoparticles } \\
\text { on mica(0001) }\end{array}$ & physisorption (drying) & $\begin{array}{l}\text { contact and tapping mode AFM for } \\
\text { imaging }\end{array}$ \\
\hline Colloidal lithography[43] & $\begin{array}{l}\text { silanol coated gold } \\
\text { nanoparticles, } 4 \mathrm{~nm}\end{array}$ & $\begin{array}{l}\text { Arrays of dot patterns on } \\
\text { silicon or glass substrates }\end{array}$ & $\begin{array}{l}\text { covalent silanol linkages } \\
\text { Si-O-Si }\end{array}$ & Tapping mode AFM for imaging \\
\hline
\end{tabular}

\section{Nanodispensing of Nanoparticles to Form Patterns}

Nanopatterns can be prepared by nanodispensing, in which a modified AFM probe is used to deposit ultra-small volumes of nanoparticle solutions onto a substrate. After the sample has been dried, the nanopatterns can be characterized with a regular AFM probe after exchanging tips. Nanodispensing, as outlined in figure $4 \mathrm{a}$, was used to deposit $25 \mathrm{~nm}$ polystyrene nanoparticles onto silicon oxide and glass substrates functionalized with aminopropyltriethoxysilane (APTES) through electrostatic interactions [44]. A drop of liquid was placed on top of a modified AFM cantilever with a channel for dispensing (Figure 4b). The channel within the AFM tip was prepared by micromachining using focused ion beam milling.

Drops of liquid were dispensed in defined arrangements by placing the tip in contact with the surface. Afterthe solvent (water) had evaporated, a defined arrangement of nanoparticle patterns was formed. After the nanosized drops of the sample had dried, an array of nanoparticle deposits was produced as shown with the example $8 \times 8$ array in figure 4c. Individual nanoparticles were resolved using tapping mode AFM characterizations, shown in the zoom-in images of figure $4 \mathrm{~d}$.
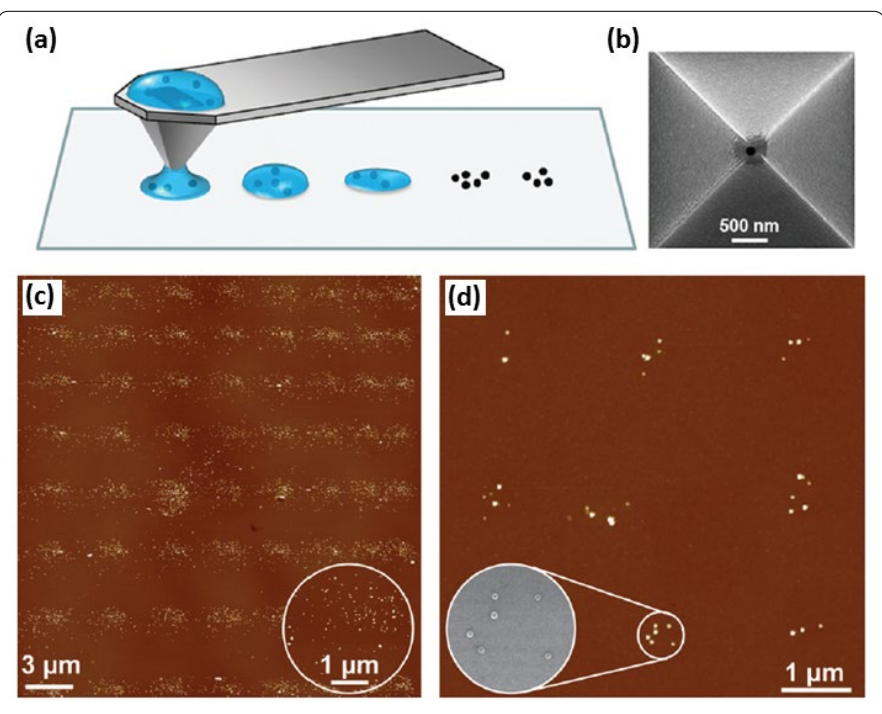

Figure 4. Nanodispensing was used to prepare an array of nanoparticles ( $25 \mathrm{~nm}$ polystyrene) on silicon oxide substrates functionalized with APTES. (a) Overview of the dispensing process;

(b) AFM tip with $130 \mathrm{~nm}$ channel viewed with SEM; (c) tappingmode AFM topograph of an array of spots prepared by nanodispensing (d) close-up AFM topography view of the nanoparticle patterns. Reprinted with permission from Ref. 44. 
As a further example of nanodispensing, gold nanoparticles $(15 \mathrm{~nm})$ were patterned on amino-functionalized silicon substrates through electrostatic interactions by Wu et al. [45] using an AFM probe fabricated with microchannels. The nanopatterns were characterized by tapping mode AFM after the liquid had evaporated. The size and height of the patterns was affected by the contact time between the tip and the substrate, for example 600-700 nm dots were produced with $2 \mathrm{~s}$ contact time. Functionalized nanodiamonds with fluorescent coatings were patterned using a "nanofountain probe" by Loh et al. [46]. The probe was designed with a reservoir to feed liquid through a microchannel to a tip with an aperture for dispensing by capillary transport. The nanodiamonds (4-10 $\mathrm{nm}$ diameter) were coated with a chemotherapeutic agent or fluorescent markers and dispensed onto glass substrates using water and dimethyl sulfoxide as solvents. Using the nanofountain probe, dot patterns were prepared with sizes ranging from $93 \mathrm{~nm}$ to 351 $\mathrm{nm}$. The patterning step was accomplished using contact mode AFM, however the ultra-small surface features were characterized with tapping mode.

\section{SPM-Based Lithography with Nanoparticles}

Innovative protocols have been developed for arranging nanoparticles on surfaces using an SPM tip as a mechanical tool for patterning. Examples of the strategies for SPM-based patterning of nanoparticles are summarized in table 3. By applying high force to the probe, local areas of films can be mechanically scratched or shaved for subsequent deposition of nanoparticles [51]. Patterns with designed interfacial chemistry can be prepared by SPM-based lithography with organic thin films to use as templates for selective deposition of nanoparticles through specific chemical interactions [12]. Dip-pen nanolithography (DPN) is another SPM-based method for patterning nanoparticles, in which an AFM tip is coated with nanoparticles to write patterns directly in defined local arrangements [39].

Table 3. Overview of Strategies Applied for SPM-Based Patterning of Nanoparticles.

\begin{tabular}{|l|l|l|l|}
\hline Method & Description & Binding Interactions & Type of nanoparticle \\
\hline Nanoshaving [52] & $\begin{array}{l}\text { An AFM tip was used to uncover selected areas of } \\
\text { substrate to form patterns }\end{array}$ & physical removal of films & $\begin{array}{l}\text { gold nanoparticles gold nanorods } \\
\text { (13-80 nm) }\end{array}$ \\
\hline Nanoshaving [51] & $\begin{array}{l}\text { Thiolated nanoparticles assembled in holes shaved } \\
\text { within an alkanethiol monolayer on Au(111) }\end{array}$ & $\begin{array}{l}\text { chemisorption } \\
\text { (S-Au) }\end{array}$ & $\begin{array}{l}\text { gold nanoparticles coated with } \\
\text { alkanethiols (8 nm) }\end{array}$ \\
\hline Tip-induced oxidation [12] & $\begin{array}{l}\text { Bias-induced oxidation with a local probe was used to } \\
\text { prepare template patterns within an organic monolayer } \\
\text { film }\end{array}$ & $\begin{array}{l}\text { electrostatic interactions } \\
\text { with amine groups }\end{array}$ & $\begin{array}{l}\text { gold nanoparticles } \\
(2-6 \mathrm{~nm})\end{array}$ \\
\hline Dip-Pen Nanolithography [39] & $\begin{array}{l}\text { The probe was coated with nanoparticles for directly } \\
\text { writing nanopatterns }\end{array}$ & $\begin{array}{l}\text { capillary diffusion from the } \\
\text { probe to sample }\end{array}$ & $\begin{array}{l}\text { gold nanoparticles } \\
(1.4-5 \mathrm{~nm})\end{array}$ \\
\hline
\end{tabular}

When applying a high mechanical force to an AFM probe, mechanical scraping or nanoshaving of surface films can be accomplished to expose small areas of the substrate. Patterns of microlines were prepared using SPM-based nanoshaving of a film of soft polymer by Chen et al. [52]. Samples were prepared by coating on $\mathrm{SiO}_{2} / \mathrm{Si}$ substrate with APTES. Negatively charged gold nanoparticles $(13 \mathrm{~nm})$ were assembled on the APTES layer through attractive electrostatic forces. A further layer of poly(methylmethacrylate) (PMMA) was spin-coated onto the surface (30 $\mathrm{nm}$ thickness). The in situ protocol for patterning with an AFM tip is shown in figure $5 a$ in which the probe was used for both steps of imaging and fabrication. An AFM tip was operated with tapping mode to scrape across the surface of the film, to produce grooves of uncovered substrate measuring $100-200 \mathrm{~nm}$ in width, as revealed with tapping mode AFM topographs in figures $5 \mathrm{~b}$ $5 \mathrm{~d}$. The 3D shape of the microchannels is shown with line profiles in the insets. At this magnification the AFM images do not clearly reveal the shapes and locations of gold nanoparticles, however electron micrographs that correspond to the samples are shown in figures $5 \mathrm{e}-5 \mathrm{~g}$. The shapes and surface density of gold nanoparticles and gold nanorods can be clearly resolved with SEM along the surface features of microlines. (b)

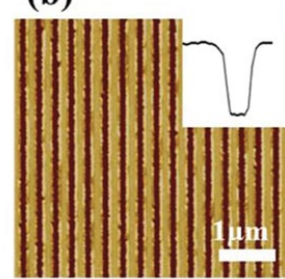

(e)

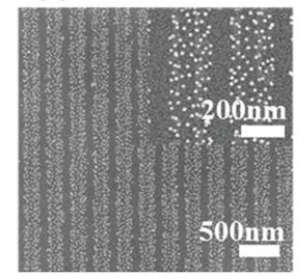

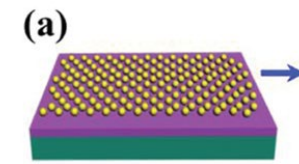

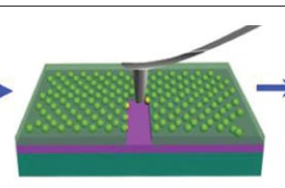

(c)

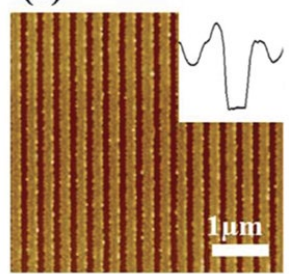

(f)

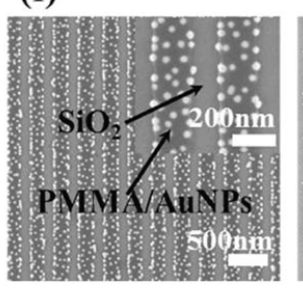

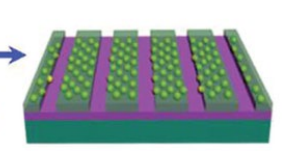

(d)

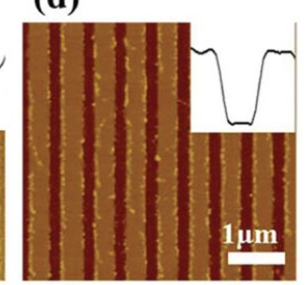

(g)

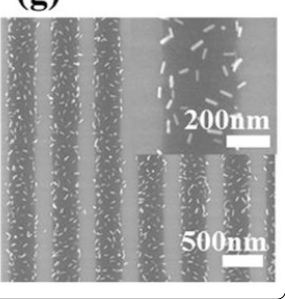

Figure 5. Microline grooves shaved within a film of APTES/

nanoparticles using an AFM tip operated in contact mode. (a) Overview of the patterning procedure. Topography frames were acquired with tapping mode in air for samples with (b) $13 \mathrm{~nm}$, (c) $33 \mathrm{~nm}$ gold nanoparticles, and (d) gold nanorods, $80 \times 20 \mathrm{~nm}$. (e-g) Scanning electron micrographs corresponding to images $b-d$. Reprinted with permission from Ref. 52. 
Dip-pen nanolithography (DPN) has been applied for direct surface patterning with nanoparticles of gold [39,53], iron oxide [38], cobalt [54], $\mathrm{CoPt}$ [55], $\mathrm{CoFe}_{2} \mathrm{O}_{4}$ [55], $\mathrm{CdS}$ [50], $\mathrm{BaFe}$ [56], and metal-organic nanocrystals [57]. The processes for patterning and imaging nanopatterns prepared with DPN were accomplished ex situ. The writing is completed using a coated probe and imaging is accomplished in a separate step using a clean tip to image patterns with either contact or tapping mode AFM. The probe for DPN is pre-coated with an ink material containing nanoparticles which diffuse onto clean surfaces when the tip is placed in direct contact with the substrate. To guide the repositioning of a clean probe, alignment marks are scored on the sample to enable subsequent characterizations of DPN nanopatterns with selected imaging modes of SPM such as contact and tapping mode. A study using the MFM mode was reported by Fu et al. for nanostructures of magnetic barium hexaferrite $\mathrm{BaFe}_{12} \mathrm{O}_{19}$, written with DPN using a tip that was coated with a sol-based ink [56]. A drawback for SPM-based methods for patterning nanoparticles is that patterns are written individually in a relatively slow, serial process. To address this problem, arrays of microfabricated probes have been developed to enable writing hundreds of patterns simultaneously using DPN [58].
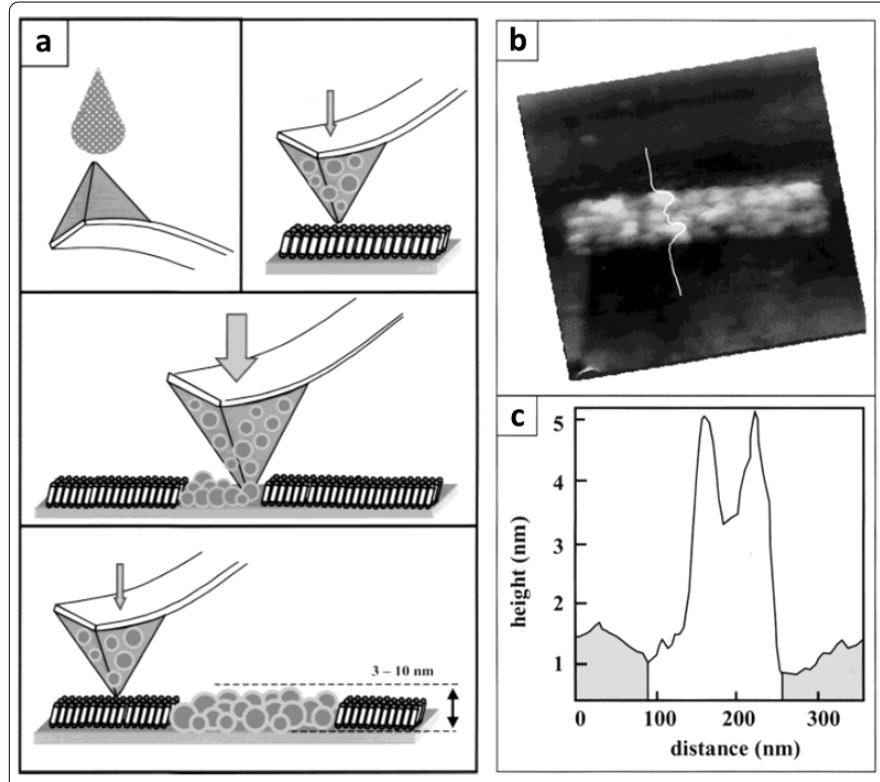

Figure 6. Nanoparticle patterning with surface active nanoparticles using SPM-based lithography. (a) Overview of the steps for in situ patterning and characterization with contact mode AFM. (b) Example pattern viewed with a contact mode topography frame. (c) Cursor profile across the pattern in $\boldsymbol{a}$. Reprinted with permission

from Ref. 51, copyright 2003 American Chemical Society.

Surface-active nanoparticles were patterned within a methyl-terminated resist layer of decanethiol using an approach based on nanoshaving [51]. The in situ procedure for patterning and characterization is shown in figure 6a. Gold nanoparticles were coated with a mixed monolayer of alkanethiol and alkanedithiol to furnish sulfur linker groups to attach to gold substrates via S-Au chemisorption. The thiolated nanoparticles physisorb to the surface of the silicon nitride AFM probe when a droplet of nanoparticle suspension is deposited and allowed to dry. When imaging in contact mode with a small force applied to the probe, the methylterminated resist surface of decanethiol/Au is not disturbed and the nanoparticles persist on the tip. When a higher force is applied to the probe, local areas of the matrix monolayer are shaved away. At the same time, with high force, the thiolated nanoparticles from the probe deposit on the gold surface following the scanning track of the tip. Returning to low force, the same probe can be used for imaging the nanopatterns. An example rectangle pattern $\left(150 \times 450 \mathrm{~nm}^{2}\right)$ of $\sim 45$ thiol-coated Au nanoparticles is shown in figure $6 \mathrm{~b}$. The height of the nanoparticles measured $4-5 \mathrm{~nm}$, shown with an example line profile in figure $6 \mathrm{c}$.

\section{Site-Directed Synthesis for Preparing Patterns of Nanoparticles}

Nanoparticles can be directly synthesized on surfaces by patterning precursor mixtures and heating the samples to complete the reaction. For example, DPN was applied to pattern metal nanoparticles $(400 \mathrm{~nm})$ on a silicon substrate using sitedirected synthesis [47]. An AFM tip (pen) was coated with a mixture of block copolymer, poly(ethylene oxide)-block-poly(2-vinyl pyridine) and metal salt (Figure 7a). Patterns were written at selected sites using DPN to deposit the polymer/metal precursor mixture to form a periodic arrangement (Figure 7b). The sample was first heated under argon at $150^{\circ} \mathrm{C}$ for annealing, which is above the glass transition temperature of the block copolymer. A second heating step at $500^{\circ} \mathrm{C}$ was then applied to decompose and remove the polymer matrix to form individual gold nanoparticles (Figure 7c). Multiple particles were formed in the absence of a thermal annealing step at lower temperature (Figure 7d).
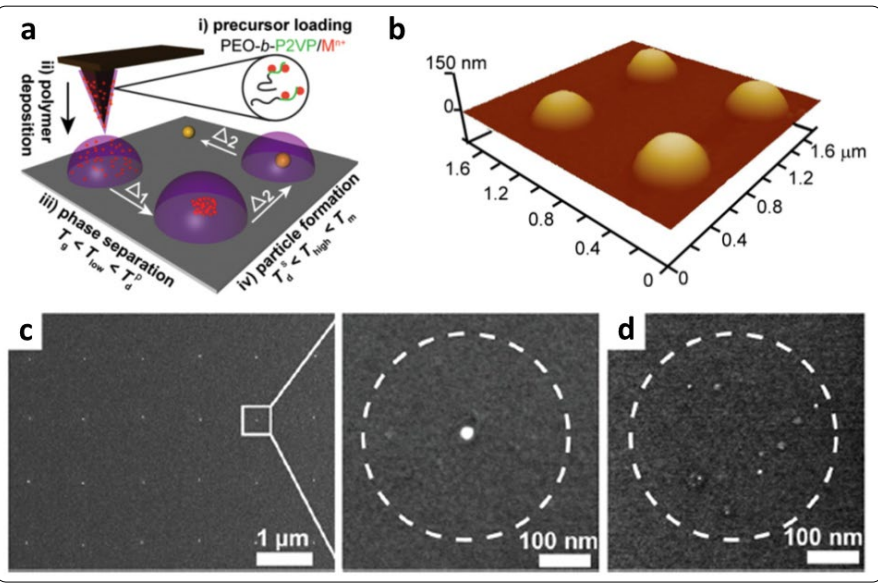

Figure 7. Site-directed synthesis of gold nanoparticles based on protocols with DPN. (a) A probe coated with precursor reagents was used to write patterns with DPN. (b) AFM topograph showing deposits of precursor written with DPN before heating. (c) After heating steps, an SEM image discloses that an array of individual gold nanoparticles $(30 \mathrm{~nm})$ was synthesized. (d) Without an annealing step, multiple nanoparticles were

formed due to diffusion. Reprinted with permission from Ref. 47.

Periodic arrays of nanoparticles composed of rare earth oxides (REO) were synthesized and patterned using protocols based on colloidal lithography as reported by Englade-Franklin et al. [9]. The synthesis of REO nanoparticles was localized specifically inside nanoholes formed within a film of octadecyl trichlorosilane (OTS) that had been prepared using a surface 
mask of $\mathrm{SiO}_{2}$ particles $(500 \mathrm{~nm})$. After an immersion step, the nanoholes within OTS were filled with a precursor solution of erbium and yttrium salts. The sample was heated to $800^{\circ} \mathrm{C}$ to form Erbium-doped $\mathrm{Y}_{2} \mathrm{O}_{3}$ nanoparticles as well as to remove the organic matrix of OTS. An array of 140 nanoparticles is disclosed in the tapping mode AFM topography frame of figure $8 \mathrm{a}$, arranged on the surface according to the periodicity $(500 \mathrm{~nm})$ of the $\mathrm{SiO}_{2}$ particles of the surface mask. The average height of the nanoparticles measured $4 \mathrm{~nm}$ as shown with an example cursor profile in figure 8b. An individual REO nanoparticle is shown in figure $8 c$, evidencing the clean removal of the OTS matrix.

(a)

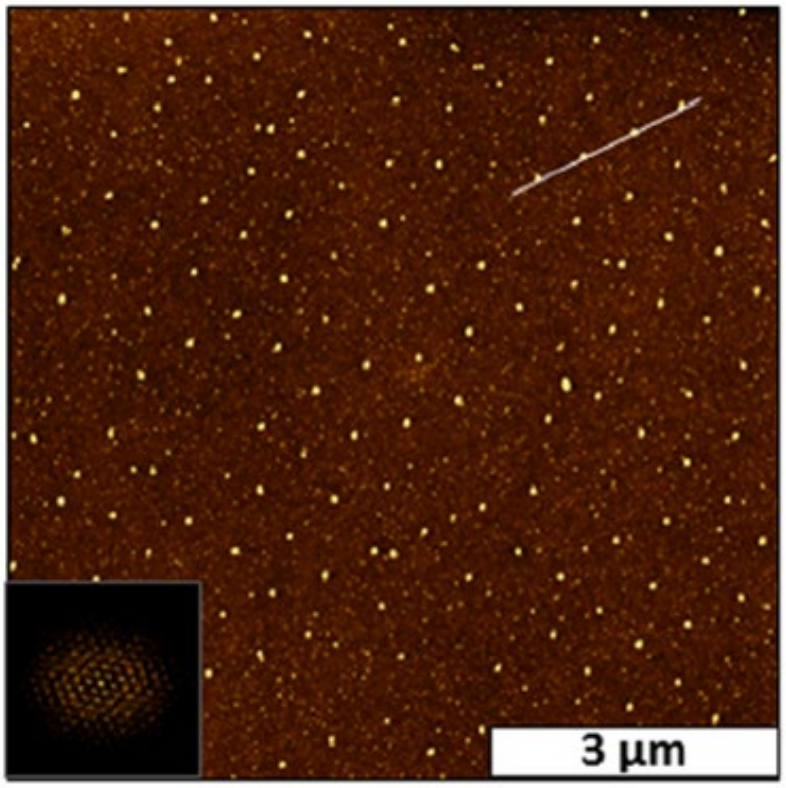

0

$3 \mathrm{~nm}$

(b)

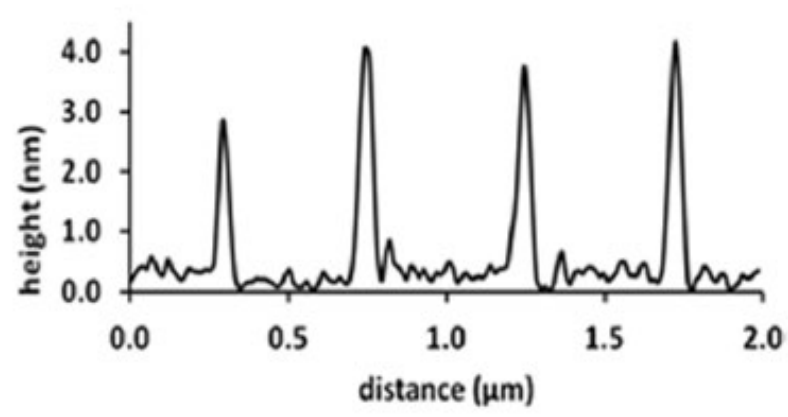

(c)

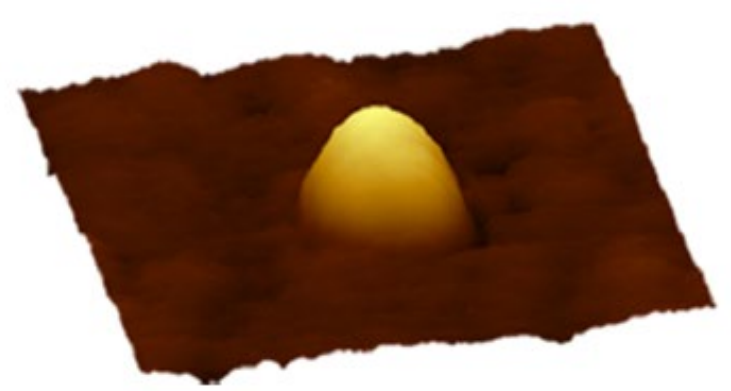

Figure 8. Site-directed synthesis of Erbium-doped $\mathrm{Y}_{2} \mathrm{O}_{3}$ nanoclusters formed on $\mathrm{Si}(111)$ after heating at $800^{\circ} \mathrm{C}$. (a) Tapping mode topograph $\left(7 \times 7 \mu \mathrm{m}^{2}\right)$ acquired in air reveals an array of REO

nanoparticles, with an FFT inset. (b) Height profile of four nanoparticles for the white line in $\boldsymbol{a}$. (c) Magnified view of a single nanoparticle. Reprinted with permission from Ref. 9.

\section{Chemically Driven Assembly of Nanoparticles on Surface Templates}

Chemically driven assembly is an approach for patterning which is based on the chemical interactions between nanoparticles and a substrate. The assembly of nanoparticles takes place on chemical patterns of polymers, sols, and selfassembled monolayers (SAMs). To bind nanoparticles, either the nanoparticles or substrate is functionalized with selected chemistries. Close-packed and regular arrays of nanoparticles have been assembled based on chemical interactions between nanoparticles and patterns of chemically specific substrates. Designing the binding chemistry for each type of particlesubstrate combination can be used to address potential problems with non-specific adsorption.

The adsorption of cationic gold nanoparticles on functionalized nanopatterns prepared by successive reaction steps was reported by Wouters and Schubert [48]. Submicronsized patterns were prepared by local probe oxidation of silicon wafers functionalized with OTS. Nanopatterns of OTS were locally oxidized using conductive AFM probes with a coating of TiN or $\mathrm{W}_{2} \mathrm{C}$ operated in contact mode $(0.5 \mathrm{~V})$. Cationic gold nanoparticles with a diameter of $20 \mathrm{~nm}$ were attached to nanopatterns functionalized with undecyltrichlorosilane through electrostatic interactions between negatively charged patterns and positively charged nanoparticlesas shown with the example of concentric ring nanopatterns in figure 9. The nanostructures of gold nanoparticles were imaged ex situ with tapping mode AFM using silicon tips.

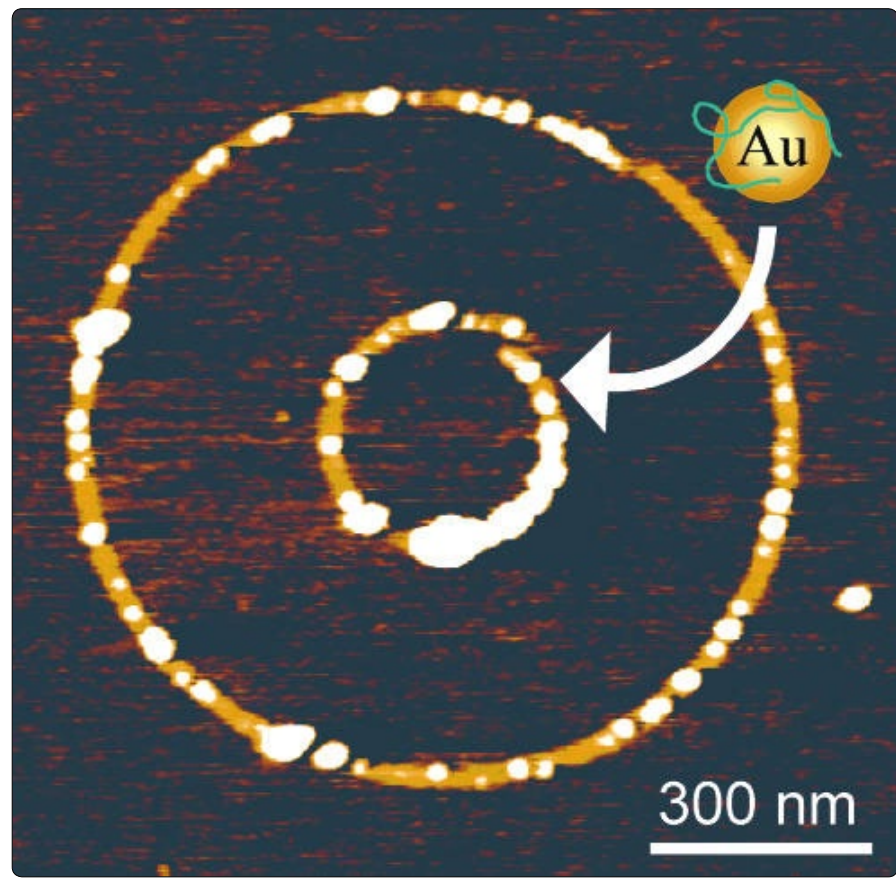

Figure 9. Cationic gold nanoparticles form a ring arrangement on oxidized OTS nanopatterns. Individual nanoparticles were resolved using tapping-mode AFM (height image, $1.1 \times 1.1 \mu \mathrm{m}, z$ range $=18$

$\mathrm{nm}$ ). Reprinted with permission from Ref. 48, copyright 2003, American Chemical Society.

Nanostructures of colloidal gold particles were patterned on silicon using a method of constructive nanolithography as reported by Liu et al. in which all of the steps were conducted 
in situ [12]. Using contact mode, an AFM instrument was configured for tip-induced local oxidation to prepare - $\mathrm{COOH}$ functionalized patterns within an inert background monolayer of methyl-terminated OTS. A $9 \times 9$ array of oxidized dots were resolved using contact mode AFM, the chemical changes are readily visualized in the friction image of figure 10a. Vinylterminated non adecenyltrichlorosilane (NTS) was then bound to the oxidized sites to form a bilayer (Figure 10b). Further steps of photoreaction and chemical reduction were used to convert the vinyl groups of NTS to amines. Gold nanoparticles (2-6 $\mathrm{nm}$ diameter) assembled selectively on the aminoterminated bilayer nanopatterns based on electrostatic interactions (Figure 10c).

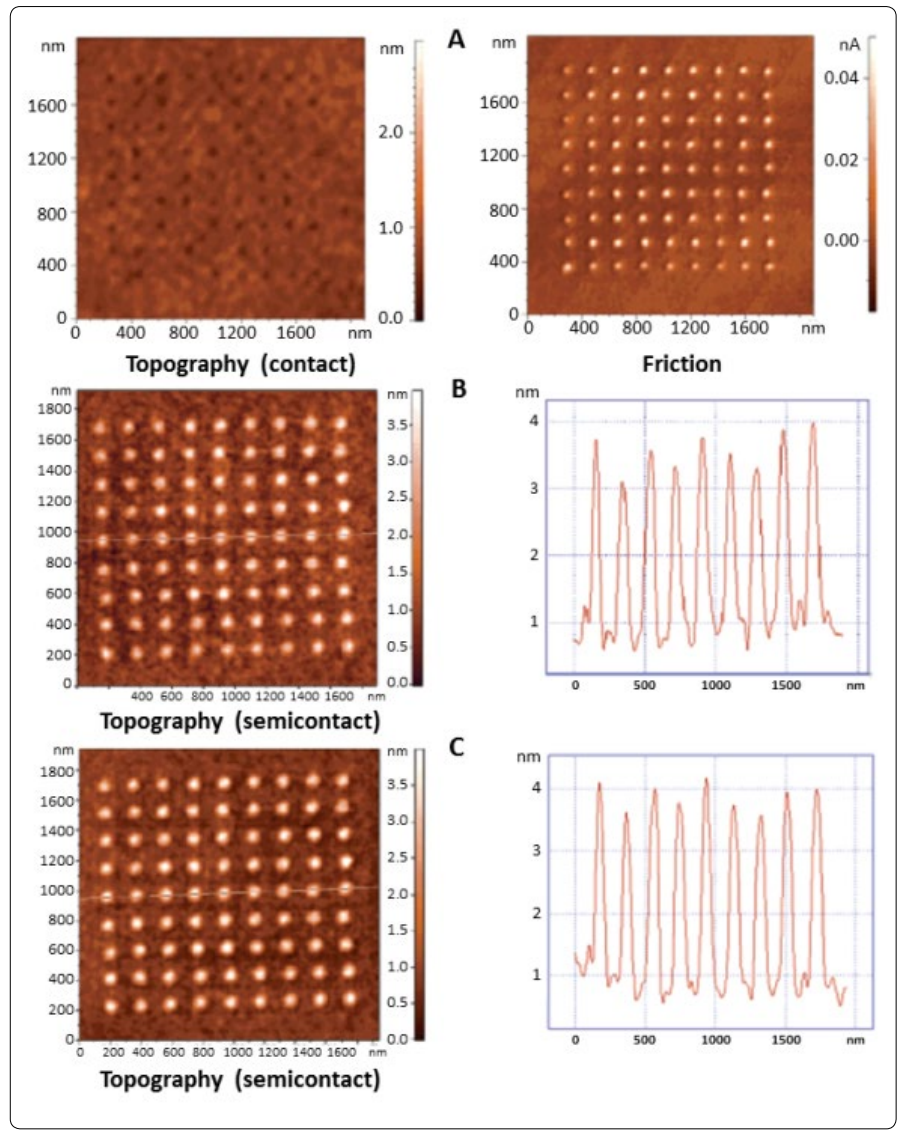

Figure 10. Surface changes mapped with modes of SPM for constructing nanopatterns of gold nanoparticles bound to NTS. (a) Contact mode images acquired after tip-induced oxidation; (b) after binding NTS selectively to the oxidized sites, tapping mode AFM was used to measure changes in height; (c) Surface changes were evaluated with tapping mode after attaching gold nanoparticles. Reprinted with permission from Ref. 12, copyright 2004, American Chemical Society.

Arrays of gold nanoparticles were prepared on indium tin oxide (ITO) with SPM-based nanoshaving of SAMs followed by chemical modification as reported by Yang et al. [59]. First an ITO substrate was coated with methyl-terminated octadecyltrimethoxysilane using heated chemical vapor deposition. In the next step, the SAM was locally worn away with AFM lithography to expose areas of the substrate by operating the AFM tip in contact mode using higher force. The exposed areas were filled with amino terminated groups by exposure to APTES vapor. Gold nanoparticles were selectively assembled on the patterned template ex situ by immersing the sample in a solution of gold nanoparticles (20 $\pm 3 \mathrm{~nm}$ ). A necklace pattern of nanoparticles was arranged to form a circle, with precise alignment of a single line of closely spaced nanoparticles.

\section{Arranging Nanoparticles on Surfaces using Colloidal Lithography}

Approaches using colloidal lithography with nanoparticles enable high throughput for preparing millions of nanopatterns with a regular periodicity defined by a surface mask of colloidal latex or silica mesoparticles. Protocols for colloidal lithography have also been referred to as nanosphere lithography or particle lithography, and implement a film of monodisperse spheres to mask local areas of the substrate. Strategies with colloidal lithography have been developed for patterning nanoparticles through chemical or physical interactions to generate circular arrangements with periodic spacing according to the dimensions of the template spheres. Colloidal lithography has been used to directly pattern nanoparticles using a film of mesospheres as a structural template, or the spheres can be used as a surface mask for patterning organic thin films which furnish reactive sites for selective binding of nanoparticles.
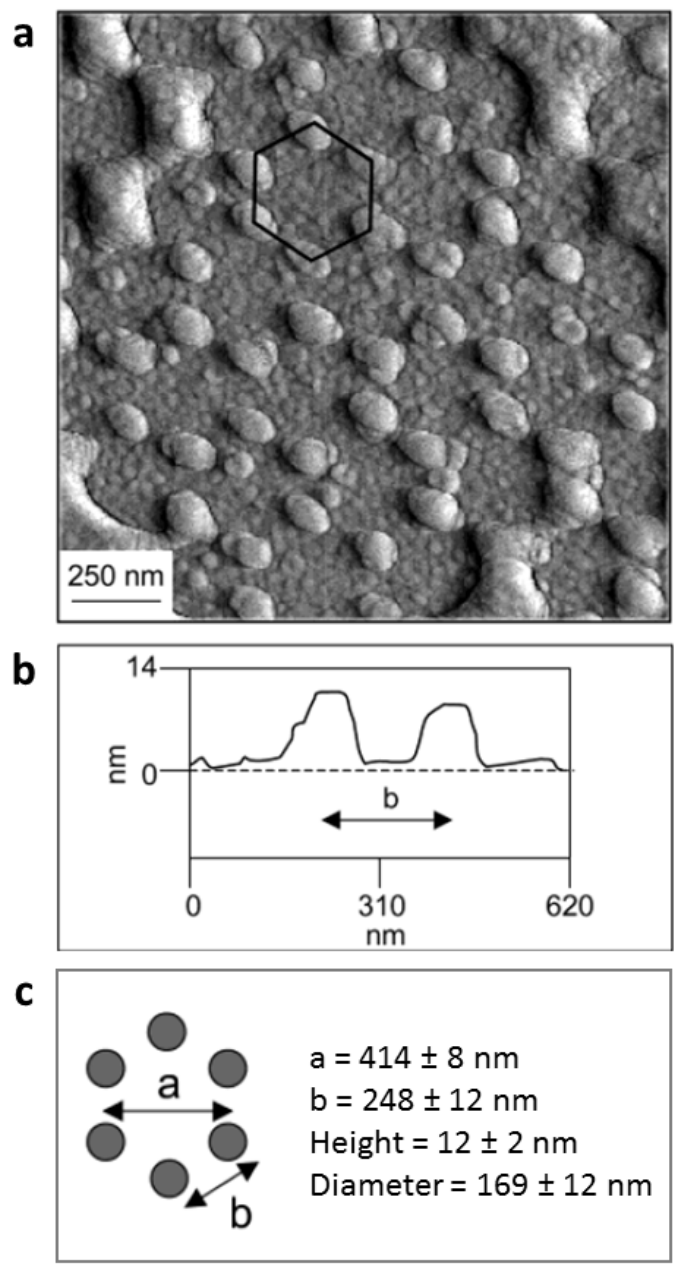

Figure 11. Hexagonal arrangement of $\mathrm{TiO}_{2}$ nanoparticles prepared on glass with colloidal lithography. (a) Topography image acquired with contact mode AFM; (b) cross-section across two $\mathrm{TiO}_{2}$ nanoparticle deposits; (c) Top-side map of the surface arrangement of $\mathrm{TiO}_{2}$ nanoparticles. Reprinted with permission from reference 49, copyright (c) 2002 American Chemical Society. 
Nanoscale lithography was used to prepare $\mathrm{TiO}_{2}$ nanoparticles by exposing a glass substrate masked with polystyrene particles to heated evaporation of titanium [49]. Metal oxide nanomaterials derived from $\mathrm{TiO}_{2}$ have been tested for applications with photocatalysis, solar energy conversion and hydrogen storage. A periodic hexagonal arrangement of $\mathrm{TiO}_{2}$ nanoclusters was deposited through the sphere mask by heating a titanium filament in a vacuum system (10-6 Torr) that was backfilled with oxygen. The surface mask was removed by sonication in ethanol to reveal an array of $\mathrm{TiO}_{2}$ particles measuring $13 \pm 2 \mathrm{~nm}$ in height as shown in figure 11a. The spacing between $\mathrm{TiO}_{2}$ deposits measured 414 $\pm 8 \mathrm{~nm}$, which follows accordingly from the $420 \mathrm{~nm}$ diameter of the sphere mask. The lattice parameters of the crystalline surface arrangement are depicted in figures $11 \mathrm{~b}$ and $11 \mathrm{c}$.
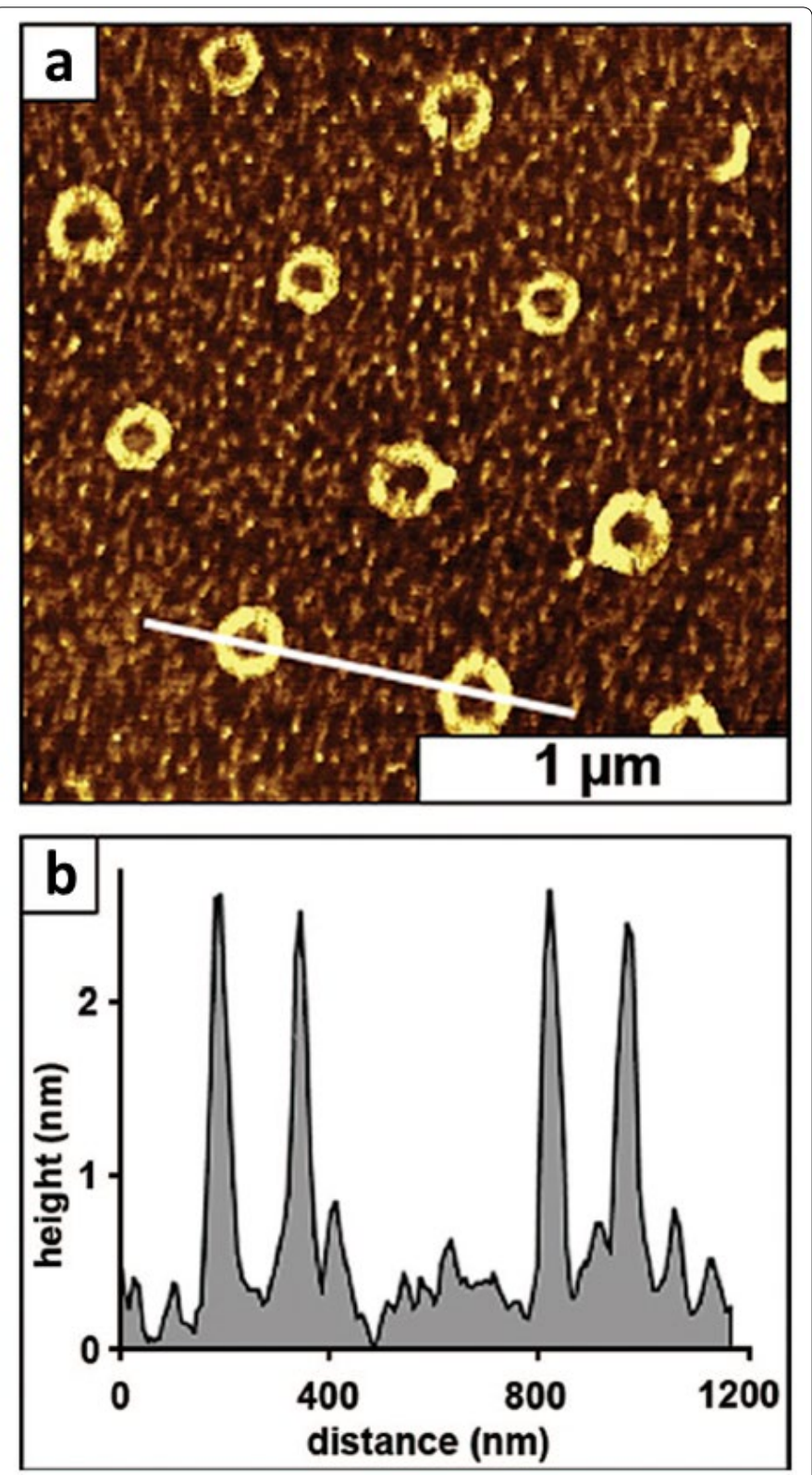

Figure 12. Two-particle lithography with cysteine-encapsulated CdS nanoparticles. (a) Tapping mode topography image of the arrangement of cysteine-encapsulated CdS nanoparticles; (b) Height cross-section for the line in $\boldsymbol{a}$ drawn across two ring patterns. Reprinted with permission from Ref. 50 copyright 2009, American Chemical Society.
An approach for "two-particle" lithography was reported by Lewandowski et al. in which particles with different sizes and composition were mixed together to generate nanopatterns [50]. Two particle lithography was used to pattern cysteine-coated CdS nanoparticles ( $2 \mathrm{~nm}$ ) on muscovite mica by evaporating a mixture of small $\mathrm{CdS}$ nanoparticles with silica spheres $(500 \mathrm{~nm})$. The particles arranged spontaneously with ambient drying so that the larger silica spheres packed together to form a surface template with a hexagonal arrangement. The CdS nanoparticles remained suspended until the solvent had fully evaporated to deposit at the meniscus sites at the base of the larger spheres. After rinsing, the larger silica spheres were completely removed because of the relatively weak adhesion between the sphere and flat substrate. With ambient drying, the smaller nanoparticles persisted on the surface to form circle patterns, as revealed in the tapping mode topograph of figure 12a. There is a hole in the center of each ring which pinpoints the location of uncovered substrate where the silica spheres had been attached. Areas between the rings contain a few bright spots which are residues of non-specifically adsorbed CdS nanoparticles and cysteine. There are 12 rings within the $2 \times 2 \mu \mathrm{m}^{2}$ area of figure $12 \mathrm{a}$, which would scale up to 300 million patterns/ $\mathrm{cm}^{2}$. Each ring contains clusters of 9-14 nanoparticles measuring $1.9 \pm 0.6 \mathrm{~nm}$ in height, which corresponds to a single layer (Figure 12b).

Arrays of organosilane nanostructures were prepared using particle lithography combined with vapor deposition of organosilanes for selective attachment of gold nanoparticles functionalized with silanol on silicon substrates, as reported by Lusker et al. [43]. Colloidal lithography was used to prepare a surface template of $200 \mathrm{~nm}$ latex spheres, which was then exposed to a heated vapor of OTS. After rinsing steps with sonication in ethanol and water, the sample with OTS patterns was immersed in a solution of gold nanoparticles $(4.5 \pm 0.4 \mathrm{~nm})$ functionalized with a coating of bifunctional mercaptopropyltrimethoxysilane (MPTMS). Surface active nanoparticles attached selectively to the uncovered sites of substrate that were exposed when the latex mask was removed. After immersion in a solution of nanoparticles, a periodic arrangement of clusters of nanoparticles is evident in the tapping mode AFM topography (Figure13a) and phase frames (Figure 13b). The methyl-terminated areas of OTS provided an effective resist, since nonspecific adsorption of nanoparticles in areas of the matrix film in between the nanopatterns were not detected. A surface model of the interfacial chemistry for the colloidal lithography protocol is shown in figure 13c. Core-shell (MPTMS-gold) nanoparticles bound selectively to exposed areas of the silicon substrate through silanol bonds, whereas the methyl-terminated areas of OTS provided a mask to prevent binding of nanoparticles. 

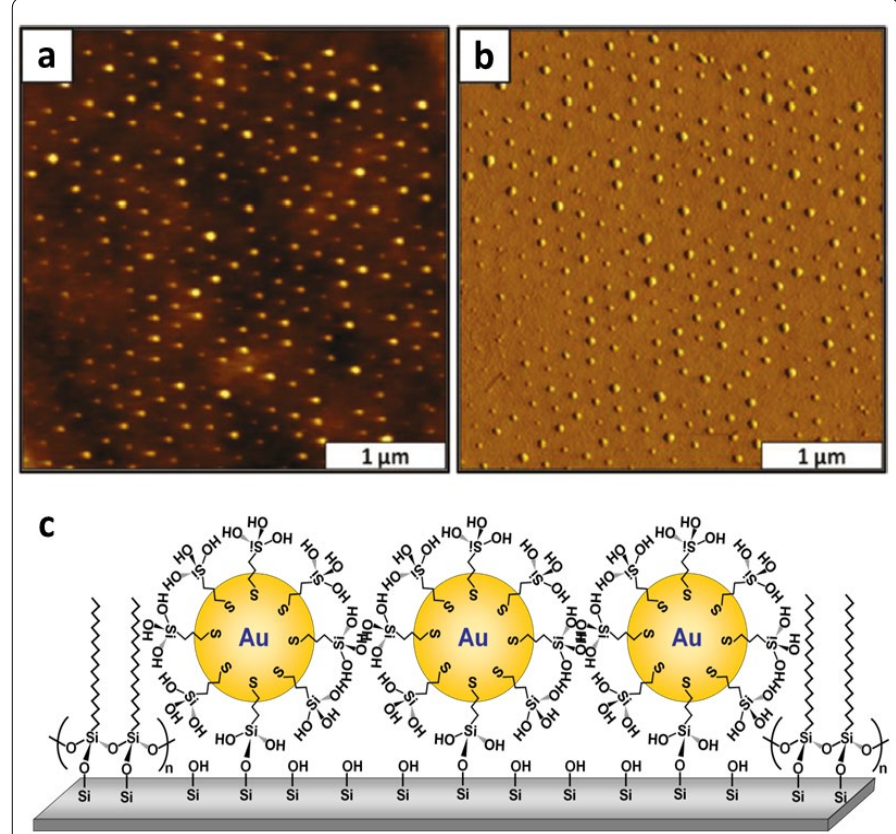

Figure 13. Array of nanopatterns prepared by lithography with organosilane encapsulated gold nanoparticles. Hexagonal arrangement of patterns of MPTMS encapsulated gold nanoparticles viewed with (a) tapping mode topography frame; (b) concurrently acquired phase image. (c) Model of the chemical interactions for binding surface active gold nanoparticles. Reprinted with permission from Ref. 43, copyright 2011, American Chemical Society.

Particle lithography was used to prepare surface patterns of core-shell nanoparticles to enable subsurface imaging with FMM as reported by Deese et al. [60]. A surface template of $500 \mathrm{~nm}$ latex spheres was prepared on silicon substrates by placing a drop $(15 \mu \mathrm{L})$ of an aqueous solution on the substrate and then drying the sample in ambient conditions. The mask of latex spheres furnished a physical template for arranging smaller nanoparticles. Next, $20 \mu \mathrm{L}$ of polystyrene-encapsulated cobalt nanoparticles (25 $\pm 3 \mathrm{~nm}$ diameter) in dichloromethane was deposited on top of the masked substrate and dried. The larger latex spheres were removed by rinsing in ethanol, while the smaller cobalt particles persisted on the surface. The nanopatterns of PS-Co nanoparticles were imaged with FMM mode to elucidate the shapes and size of the inner cobalt core underneath the polymer coating (Figure 14). A circular arrangement of nanoparticles is revealed in the topography frame of Figure 14a. The center of the rings indicates the sites of the template latex spheres. The concurrently acquired amplitude (Figure 14b) and phase (Figure 14c) channels provide information about the elastic properties of the sample. A magnified view (Figure $14 d-14 f)$ of six nanoparticles reveals differences in material composition. The amplitude and phase images disclose three distinct regions for the sample, revealing differences in contrast for the substrate, polymer shell, and inner cobalt core. The brighter regions indicate the (hard) cobalt metal centers, the intermediate color maps areas of the substrate, the darkest colored are as reveal the softer polymer coating of the nanoparticles.

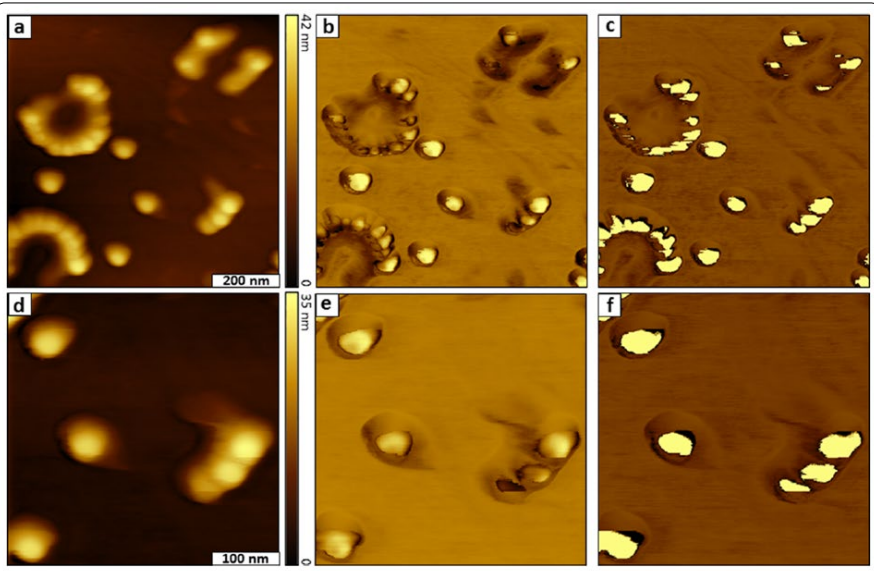

Figure 14. Images of ring nanopatterns of core-shell nanoparticles acquired with FMM mode. (a) Topograph $\left(800 \times 800 \mathrm{~nm}^{2}\right)$ acquired with FMM; (b) simultaneously acquired amplitude, and (c) phase images. (d) Close-up topography view with FMM $\left(400 \times 400 \mathrm{~nm}^{2}\right)$; (e) corresponding amplitude and (f) phase frames reveal cobalt cores encapsulated within a polymer coating. Reprinted with permission from Ref. 60, copyright 2017, American Chemical Society.
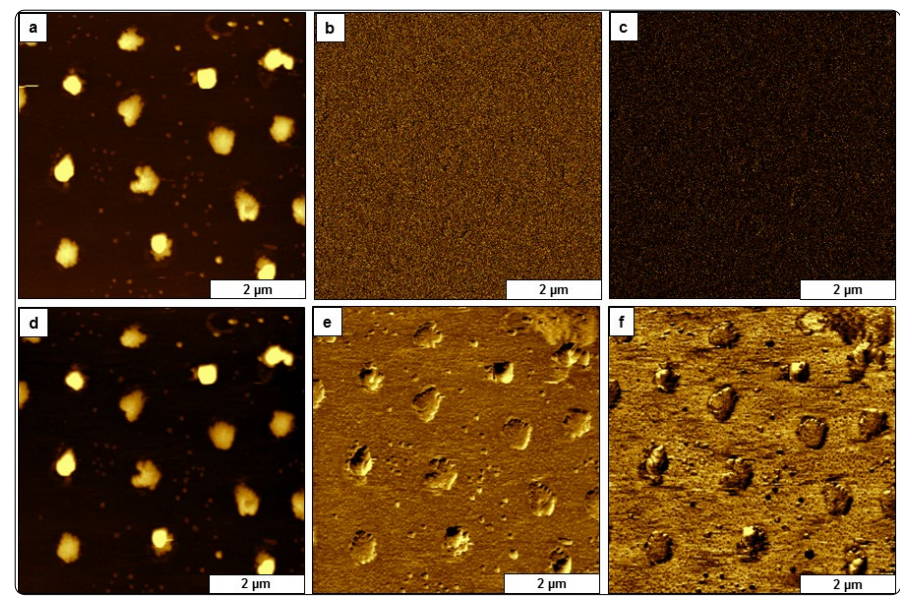

Figure 15. Colloidal lithography was used to prepare patterns of $\mathrm{Fe}_{3} \mathrm{O}_{4}$ nanoparticles. Top row: (a) Topography; (b) amplitude, and (c) phase channels were imaged without an applied magnetic field. Bottom row: (d) MSM-topography; (e) MSM-amplitude, and (f) MSM-phase frames detect the vibration of magnetic nanoparticles. Reprinted with permission from Ref. 33.

Nanopatterns of iron oxide nanoparticles $\left(\mathrm{Fe}_{3} \mathrm{O}_{4}\right)$ were prepared with a protocol of two particle lithography and characterized with MSM-AFM, by Kulkarni et al. [33]. A simple chemical strategy with basic steps of mixing, drop-deposition, drying and removing the surface template of latex spheres was used to accomplish colloidal lithography. The templating latex spheres $1 \mu \mathrm{m}$ in diameter were mixed directly with smaller $\mathrm{Fe}_{3} \mathrm{O}_{4}$ nanoparticles and deposited on glass substrates. After drying, the larger spheres of latex were physically removed by pressing a piece of adhesive tape on to the sample and pulling off the latex film. The metal nanoparticles assembled spontaneously in areas surrounding the latex template to form a periodic arrangement of clusters, with a few individual nanoparticles scattered in areas between the aggregates revealed in the MSM-topography frame of figure 15a. With the MSM mode, when no electromagnetic field was applied to the sample, there were no distinguishable features resolved in the corresponding MSM-amplitude (Figure 15b) 
and MSM-phase (Figure 15c) frames. When an AC electromagnetic field was applied (Figures 15d-15f), the amplitude and phase channels reveal sensitive maps of the magnetic areas of the sample.

\section{Conclusions}

The capabilities for preparing distinct, well-defined nanopatterns comprised of nanoparticles was reviewed along with strategies for subsequent measurements with SPM. To fully exploit the size-dependent quantum properties of metal nanoparticles, approaches for preparing defined arrangements of metal nanoparticles on surfaces with robust chemical or physical attachment are essential for the design and manufacture of next-generation devices. Beyond traditional applications of SPM for imaging, the capabilities for SPM-based nanofabrication were evaluated for studying nanoparticles. There are myriad possibilities for combining scanning probe microscopy techniques and nanoscale lithography for surface platforms used in sensors and electronic devices based on nanoparticles.

\section{Notes}

The authors declare no competing financial interest.

\section{Acknowledgments}

The authors gratefully acknowledge financial support from the Louisiana Board of Regents Support Fund, Traditional Enhancement Program (LEQSF(2014-16)-ENH-TR-03) and from Louisiana State University.

\section{References}

1. Hulteen JC, Treichel DA, Smith MT, Duval ML, Jensen TR, Van Duyne RP. Nanosphere Lithography: Size-Tunable Silver Nanoparticle and Surface Cluster Arrays. J Phys Chem B. 1999; 103: 3854-3863. doi: 10.1021/jp9904771

2. Shipway AN, Katz E, Willner I. Nanoparticle Arrays on Surfaces for Electronic, Optical and Sensor Applications. Chem Phys Chem. 2000; 1(1): 18-52. doi: 10.1002/1439-7641(20000804)1:1<18::AID-CPHC18>3.0.CO;2-L

3. Minari T, Liu X, Kanehara M. Printable Electronic Circuits Based on Metal Nanoparticles and Organic Semiconductors. International Conference on Electronics Packaging and iMAPS All Asia conference (ICEP-IAAC); 2018. doi: 10.23919/ICEP.2018.8374671

4. Li Y, Wu Y, Ong BS. Facile Synthesis of Silver Nanoparticles Useful for Fabrication of High-Conductivity Elements for Printed Electronics. J Am Chem Soc. 2005; 127(10): 3266-3267.

5. Li Q, Zheng J, Liu Z. Site-Selective Assemblies of Gold Nanoparticles on an AFM Tip-Defined Silicon Template. Langmuir. 2003; 19: 166-171. doi: 10.1021/la0259149

6. Shawrav MM, Taus P, Wanzenboeck HD, et al. Highly Conductive and Pure Gold Nanostructures Grown by Electron Beam Induced Deposition. Sci Rep. 2016; 6: 34003. doi: 10.1038/srep34003

7. Zhang J, Li X, Liu K, et al. Thin Films of Ag Nanoparticles Prepared from the Reduction of AgI Nanoparticles in Self-Assembled Films. J Colloid Interface Sci. 2002; 255(1): 115-118. doi: 10.1006/jcis.2002.8615

8. Liu H, Schwenke AM, Kretschmer F, Stephanie Hoeppener, Schubert US. Gold Nanoparticle Cluster Arrays for High-Performance SERS Substrates Fabricated by Electro-oxidative Lithography. ChemNanoMat. 2016; 2(8): 781-785. doi: 10.1002/cnma.201600063

9. Englade-Franklin LE, Morrison G, Verberne-Sutton SD, Francis AL, Chan JY, Garno JC. Surface-Directed Synthesis of Erbium-Doped Yttrium Oxide Nanoparticles within Organosilane Zeptoliter Containers. ACS Appl Mater Interfaces. 2014; 6(18): 15942-15949. doi: 10.1021/am503571z
10. Zhang H, Cadusch J, Kinnear C, James T, Roberts A, Mulvaney P. Direct Assembly of Large Area Nanoparticle Arrays. ACS Nano. 2018; 12(8): 7529-7537.

11. Kelley $A T$, Serem WK, Daniels $\mathrm{SL}$, et al. Vibrational Response of $\mathrm{FeNi}_{3}$ Nanoparticles to the Flux of a Modulated Electromagnetic Field Detected by Contact-Mode Atomic Force Microscopy. J Phys Chem C. 2013; 117(36): 18768-18776. doi: 10.1021/jp406919n

12. Liu S, Maoz R, Sagiv J. Planned Nanostructures of Colloidal Gold via SelfAssembly on Hierarchically Assembled Organic Bilayer Template Patterns with in-situ Generated Terminal Amino Functionality. Nano Lett. 2004; 4 845-851. doi: $10.1021 / \mathrm{nl} 049755 \mathrm{k}$

13. Radmacher M, Tillmann RW, Gaub HE. Imaging Viscoelasticity by Force Modulation with the Atomic Force Microscope. Biophys J. 1993; 64(3): 735-742. doi: 10.1016/S0006-3495(93)81433-4

14. Hansma PK, Elings VB, Marti O, Bracker CE. Scanning Tunneling Microscopy and Atomic Force Microscopy: Application to Biology and Technology. Science. 1988; 242(4876): 209-216.

15. Binnig G, Gerber C, Stoll E, Albrecht TR, Quate CF. Atomic Resolution with Atomic Force Microscope. Europhys Lett. 1987; 3(12): 1281.

16. Albrecht TR, Quate CF. Atomic Resolution Imaging of a Nonconductor by Atomic Force Microscopy. J Appl Phys. 1987; 62(7): 2599-2602.

17. Barth C, Henry CR. Atomic Resolution Imaging of the (001) Surface of UHV Cleaved MgO by Dynamic Scanning Force Microscopy. Phys Rev Lett. 2003; 91(19): 196102. doi: 10.1103/PhysRevLett.91.196102

18. Gratz AJ, Manne S, Hansma PK. Atomic Force Microscopy of Atomic-Scale Ledges and Etch Pits Formed During Dissolution of Quartz. Science. 1991; 251(4999): 1343-1346

19. Lauritsen JV, Reichling M. Atomic Resolution Non-Contact Atomic Force Microscopy of Clean Metal Oxide Surfaces. J Phys: Condens Matter. 2010; 22(26): 263001. doi: 10.1088/0953-8984/22/26/263001

20. Maivald P, Butt HJ, Gould SAC, et al. Using Force Modulation to Image Surface Elasticities with the Atomic Force Microscope. Nanotechnology. 1991; 2(2): 103.

21. Li F-B, Thompson GE, Newman RC. Force Modulation Atomic Force Microscopy: Background, Development and Application to Electrodeposited Cerium Oxide Films. Appl Surf Sci. 1998; 126(1-2): 21-33. doi: 10.1016/S0169-4332(97)00590-4

22. DeVecchio D, Bhushan B. Localized Surface Elasticity Measurements Using an Atomic Force Microscope. Rev Sci Instrum. 1997; 68(12): 44984505. doi: $10.1063 / 1.1148420$

23. Piétrement O, Troyon M. Quantitative Elastic Modulus Measurement by Magnetic Force Modulation Microscopy. Tribol Lett. 2000; 9(1): 77-87. doi: 10.1023/A:1018860528388

24. Straka L, Fekete L, Heczko O. Antiphase Boundaries in Bulk Ni-Mn-Ga Heusler Alloy Observed by Magnetic Force Microscopy. Appl Phys Lett. 2018; 113(17): 172901. doi: 10.1063/1.5052408

25. Cao Y, Kumar P, Zhao Y, Suzuki Y, Yoshimura S, Saito H. High Magnetization Co-GdOx Superparamagnetic Granular Films as Magnetic Coating Materials for High-Sensitivity Alternating Magnetic Force Microscopy Tip. J Magn Magn Mater. 2018; 462(15): 119-126.

26. Li X, Lu W, Song Y, et al. Quantitatively Probing the Magnetic Behavior of Individual Nanoparticles by an AC Field-Modulated Magnetic Force Microscopy. Sci Rep. 2016; 6: 22467.

27. Clendenning SB, Fournier-Bidoz S, Pietrangelo A, et al. Ordered 2D Arrays of Ferromagnetic Fe/Co Nanoparticle Rings from a Highly Metallized Metallopolymer Precursor. J Mater Chem. 2004; 14: 1686-1690. doi: 10.1039/B402027H

28. Hamann HF, Woods SI, Sun S. Direct Thermal Patterning of Self-Assembled Nanoparticles. Nano Lett. 2003; 3: 1643-1645. doi: 10.1021/nl034706d

29. Jourdain $V$, Simpson ET, Paillet $M$, et al. Periodic Inclusion of RoomTemperature-Ferromagnetic Metal Phosphide Nanoparticles in Carbon Nanotubes. J Phys Chem B. 2006; 110(20): 9759-9763.

30. Netto CG, Nakamatsu EH, Netto LE, et al. Catalytic Properties of Thioredoxin Immobilized on Superparamagnetic Nanoparticles. J Inorg Biochem. 2011; 105(5): 738-744. doi: 10.1016/j.jinorgbio.2011.02.006 
31. Daniels SL, Ngunjiri JN, Garno JC. Investigation of the Magnetic Properties of Ferritin by AFM Imaging with Magnetic Sample Modulation. Anal Bioanal Chem. 2009; 394(1): 215-223. doi: 10.1007/s00216-009-2618-y

32. Li J-R, Lewandowski BR, Xu S, Garno JC. Detecting the Magnetic Response of Iron Oxide Capped Organosilane Nanostructures Using Magnetic Sample Modulation and Atomic Force Microscopy. Anal Chem. 2009; 81(12): 4792-4802. doi: 10.1021/ac900369v

33. Kulkarni SA, Kolhatkar AG, Lee TR, Garno JC. Vibrational Response of Clusters of $\mathrm{Fe}_{3} \mathrm{O}_{4} \mathrm{Nanoparticles} \mathrm{Patterned} \mathrm{on} \mathrm{Glass} \mathrm{Surfaces} \mathrm{Investigated}$ with Magnetic Sample Modulation AFM. Nanoscale. 2018;10(43):2042620434.

34. Chu C, Na JS, Parsons GN. Conductivity in Alkylamine/Gold and Alkanethiol/Gold Molecular Junctions Measured in Molecule/ Nanoparticle/Molecule Bridges and Conducting Probe Structures. J Am Chem Soc. 2007; 129(8): 2287-2296.

35. Cho SH, Park SM. Electrochemistry of Conductive Polymers 39. Contacts between Conducting Polymers and Noble Metal Nanoparticles Studied by Current-Sensing Atomic Force Microscopy. J Phys Chem B. 2006; 110(51): 25656-25664. doi: 10.1021/jp0656781

36. Grzelczak M, Vermant J, Furst EM, Liz-Marzan LM. Directed Self-Assembly of Nanoparticles. ACS Nano. 2010; 4: 3591-3605. doi: 10.1021/nn100869j

37. Hermanson KD, Lumsdon SO, Williams JP, Kaler EW, Velev OD. Dielectrophoretic Assembly of Electrically Functional Microwires from Nanoparticle Suspensions. Science. 2001; 294: 1082-1086. doi: 10.1126/ science. 1063821

38. Liu X, Fu L, Hong S, Dravid VP, Mirkin CA. Arrays of Magnetic Nanoparticles Patterned via "Dip-Pen" Nanolithography. Adv Mater. 2002; 14(3): 231234.

39. Wang WM, Stoltenberg RM, Liu $S$, Bao Z. Direct Patterning of Gold Nanoparticles Using Dip-Pen Nanolithography. ACS Nano. 2008; 2(10): 2135-2142. doi: $10.1021 / n n 8005416$.

40. LiX-M, Paraschiv V, Huskens J, Reinhoudt DN. Sulfonic Acid-Functionalized Gold Nanoparticles: A Colloid-Bound Catalyst for Soft Lithographic Application on Self-Assembled Monolayers. J Am Chem Soc. 2003; 125(14): 4279-4284. doi: 10.1021/ja0271431

41. Chan JC, Hannah-Moore N, Rananavare SB. Controlled Deposition of Tin Oxide and Silver Nanoparticles Using Microcontact Printing. Crystals. 2015; 5(1): 116-142.

42. Wang Y, Han S, Briseno AL, Sanedrin RJG, Zhou F. A Modified Nanosphere Lithography for the Fabrication of Aminosilane/Polystyrene Nanoring Arrays and the Subsequent Attachment of Gold or DNA-Capped Gold Nanoparticles.J Mater Chem. 2004; 14: 3488-3494. doi: 10.1039/B406997H

43. Lusker KL, Li J-R, Garno JC. Nanostructures of Functionalized Gold Nanoparticles Prepared by Particle Lithography with Organosilanes. Langmuir. 2011; 27: 13269-13275.

44. Fabié $L$, Agostini $P$, Stopel $M$, et al. Direct patterning of nanoparticles and biomolecules by liquid nanodispensing. Nanoscale. 2015; 7(10): 44974504. doi: $10.1039 / \mathrm{c} 4 \mathrm{nr} 06824 \mathrm{f}$

45. Wu B, Ho A, Moldovan N, Espinosa HD. Direct Deposition and Assembly of Gold Colloidal Particles Using a Nanofountain Probe. Langmuir 2007; 23(17): 9120-9123. doi: 10.1021/la7011952
46. Loh O, Lam R, Chen M, et al. Nanofountain-Probe-Based High-Resolution Patterning and Single-Cell Injection of Functionalized Nanodiamonds. Small. 2009; 5(14): 1667-1674. doi: 10.1002/smll.200900361

47. Liu G, Eichelsdoerfer DJ, Rasin B, et al. Delineating the Pathways for the Site-Directed Synthesis of Individual Nanoparticles on Surfaces. Proc Natl Acad Sci USA. 2013; 110: 887-891. doi: 10.1073/pnas.1220689110

48. Wouters D, Schubert US. Constructive Nanolithography and Nanochemistry: Local Probe Oxidation and Chemical Modification. Langmuir 2003; 19: 9033-9038. doi: 10.1021/la034711x

49. Bullen $\mathrm{HA}$, Garrett $\mathrm{SJ}$. $\mathrm{TiO}_{2}$ Nanoparticle Arrays Prepared Using a Nanosphere Lithography Technique. Nano Lett. 2002; 2: 739-745. doi: $10.1021 / \mathrm{nl} 025568 \mathrm{f}$

50. Lewandowski BR, Kelley AT, Singleton R, et al. Nanostructures of CysteineCoated CdS Nanoparticles Produced with "Two-Particle" Lithography. J Phys Chem C. 2009; 113: 5933-5940. doi: 10.1021/jp808056x

51. Garno JC, Yang Y, Amro NA, Cruchon-Dupeyrat S, Chen S, Liu G-Y. Precise Positioning of Nanoparticles on Surfaces Using Scanning Probe Lithography. Langmuir. 2003; 3: 389-395. doi: 10.1021/n1025934v

52. Chen J, Sun Y, Zhong L, et al. Scalable Fabrication of Multiplexed Plasmonic Nanoparticle Structures Based on AFM Lithography. Small. 2016; 12(42): 5818-5825. doi: 10.1002/smll.201602250

53. Ali MB, Ondarçuhu T, Brust M, Joachim C. Atomic Force Microscope Tip Nanoprinting of Gold Nanoclusters. Langmuir. 2002; 18: 872-876. doi: 10.1021/la011023+

54. Bellido $E$, Ojea-Jiménez I, Ghirri A, et al. Controlled Positioning of Nanoparticles on Graphene by Noninvasive AFM Lithography. Langmuir 2012; 28(33): 12400-12409. doi: 10.1021/la3023419

55. Roy $D$, Munz $M$, Colombi $P$, et al. Directly Writing with Nanoparticles at the Nanoscale Using Dip-Pen Nanolithography. Appl Surf Sci. 2007; 254(5): 1394-1398. doi: 10.1016/j.apsusc.2007.06.058

56. Fu L, Liu X, Zhang Y, Dravid VP, Mirkin CA. Nanopatterning of "Hard" Magnetic Nanostructures via Dip-Pen Nanolithography and a Sol-Based Ink. Nano Lett. 2003; 3: 757-760.

57. Bellido E, Cardona-Serra S, Coronado E, Ruiz-Molina D. Assisted-Assembly of Coordination Materials into Advanced Nanoarchitectures by Dip Pen Nanolithography. Chem Commun. 2011; 47(18): 5175-5177.

58. Salaita K, Wang Y, Fragala J, Vega RA, Liu C, Mirkin CA. Massively Parallel Dip-Pen Nanolithography with 55000-Pen Two-Dimensional Arrays. Angew Chem Int Ed Engl. 2006; 45(43): 7220-7223. doi: 10.1002/ anie.200603142

59. Yang J, Ichii T, Murase K, Sugimura H. Circular Arrays of Gold Nanoparticles of a Single Particle Line Thickness Formed on Indium Tin Oxide. Appl Phys Express. 2012; 5: 025202. doi: 10.1143/APEX.5.025202

60. Deese SM, Englade-Franklin LE, Hill L, Pyun J, Chan JY, Garno JC. Subsurface Imaging of the Cores of Polymer-Encapsulated Cobalt Nanoparticles Using Force Modulation Microscopy. J Phys Chem C. 2017 121: 23498-23504. doi: 10.1021/acs.jpcc.7b07994 